\title{
Infrastructuring for Cultural Commons
}

\author{
Sanna Marttila 1 (1) \& Andrea Botero ${ }^{2}$ \\ ${ }^{1}$ School of Arts, Design and Architecture, Aalto University, P.O.Box 16500, Espoo, FI-00076 Aalto, \\ Finland (E-mail: sanna.marttila@aalto.fi); ${ }^{2}$ Faculty of Architecture and Design, Universidad de Los \\ Andes, Cra $1 N^{\circ} 18 A$ - 12, Bogotá, Colombia (E-mail: andrea.botero@iki.fi)
}

\begin{abstract}
In this paper we reflect on our involvement in the design and development of two information systems: Fusion and EUscreen. Both are infrastructural initiatives aimed at contributing, from different angles, to wider public access to and appropriation of the European digital cultural heritage. Our analysis is founded on the notions of an installed base and gateway in information infrastructure development. We situate our co-design activities and infrastructuring strategies in relation to a broader interest in advocating not only the preservation of and access to digital cultural heritage, but, more importantly, enabling collaboration, to support the emerging practices of diverse user groups, and to contribute to cultural commons.
\end{abstract}

Keywords: Infrastructuring, Co-design, Commons, Cultural heritage, Practice

\section{Introduction}

In recent decades, European countries and their cultural and memory institutions (e.g. libraries, archives and museums) have invested heavily in efforts to digitize cultural heritage content by creating digital reproductions and copies of cultural artefacts, such as documents, paintings and audiovisual materials. ${ }^{1}$ Increasingly large-scale conservation and digitization initiatives are building infrastructures for archiving cultural heritage in digital forms, and creating access to this common, shared history and culture. For institutions these digitalization initiatives create potentials for the long-term preservation of their collections, as well as for expanding public access to and use of their holdings (for examples of initiatives see (Sotirova et al. 2012). Inviting people to interact with these growing digital cultural heritage collections enables new channels and means for enjoying and experiencing culture and history, and potentially encourages cultural appropriation and creative re-use of these collections in various sectors of society.

\footnotetext{
${ }^{1}$ It should be noted that 'cultural heritage' or 'memory institution' are not unproblematic terms, and controversies have arisen both regarding the representation of memory, and about what counts as heritage and historical materials and what does not. In this article we refer to memory institutions as being contested, complex sites that preserve and care for a repository of public knowledge, and which shape cultural meanings and understandings through various processes of knowledge production. According to (Stainforth 2016) the term memory institution has been developed over the past 20 years to describe entities that are connected through digital technologies and media, and denotes cultural integration across nations.
} 
Despite these efforts to increase open access to digital cultural heritage, today, only a small fraction of the digital holdings in Europe are being made accessible to the wider public by cultural institutions (Stroeker and Vogels 2014). In cases where digital cultural heritage materials have been made accessible online, they are often released under restrictive terms of use (Bellini et al. 2014; Estermann et al. 2015; Estermann 2015) and the scope for circulation and collaborative re-use of digital heritage is often limited (Terras 2015; Marttila and Hyyppä 2014a). As argued by many scholars (Tsolis et al. 2011; Anderson 2013), copyrights and other rights issues are an important factor preventing democratic access to and use of our digital cultural heritage. Intellectual property rights and privacy issues limit institutions in multiple ways - it is often the case that either the institutions do not hold the rights to everything in their collections, or they do not have the resources to conduct the process of clearing the rights. Commentators also point out that many culture and memory institutions fear a loss of authority and control over their collections, or fear losing possible sources of future revenue if they release digital cultural materials on more open terms (Tsolis et al. 2011; Verwayen et al. 2011).

Along with these institutional efforts to digitize cultural heritage and create online access to collections, an abundance of digital tools and platforms have been created to stimulate and support so-called amateur and peer-to-peer efforts to access, appropriate and reinvent culture (Bruns 2008; Jenkins 2006; Bauwens 2009). Due to these infrastructures that mediate digital culture, and the concomitant socio-technical media practices, today, large groups of people and communities are engaged in a variety of cultural-production practices that both create new forms of digital cultural heritage and, at the same time, circulate older forms of heritage in novel ways. These rich infrastructures encompass everything from hobbyist communities that document their own cultural practices and content in commercial settings, such as Instructables, Flickr or Pinterest, to community efforts that use infrastructures such as Wikipedia and Wikimedia Commons to create alternative cultural heritage repositories (e.g. the Wiki Loves Monuments initiative, and the project on Public Art on Wikipedia), to general social-media platforms that are arenas for new forms of digital culture (e.g. animated GIFs, memes).

We believe there is great potential in fostering a productive, collaborative relationship between, on the one hand, institutionalized digital cultural heritage preservation initiatives, and, on the other hand, amateur and peer-to-peer online media practices and infrastructures. Moreover, we are interested in acknowledging digital cultural heritage infrastructures as being not only concerned with preservation and access to digital cultural heritage, but also as important catalysts in the construction of shared cultural resources that are equally concerned with enabling collaboration between a diversity of audiences. In order to address these broader interests in this paper we reflect on our involvement in the design and development of two infrastructural initiatives aimed at contributing, from different angles, to wider public access to and cultural appropriation and re-use of European digital and audiovisual cultural heritage. The first infrastructure, named Fusion, aimed to develop a peer-to- 
peer audiovisual file-sharing system for creative communities and their emerging media practices. The second initiative, EUscreen, developed a Europe-wide portal for digital audiovisual heritage.

To frame these two cases we build on the concept of commons, understood as particular arrangements for managing and governing shared resources (e.g. Ostrom and Hess 2007; Benkler 2013; Bollier and Helfrich 2012). Our focus is particularly on the characteristics of the concept that has lately been referred to as cultural commons (Madison et al. 2010; Hyde 2010; Hess 2012; Bertacchini et al. 2012). To analyse these cases we combine this broader framing of commons with a discussion of the concepts of infrastructure and infrastructuring processes, as being central to contemporary discussions of design (Star and Bowker 2002; Karasti 2014).

Research on the collaborative design of infrastructures for digital cultural heritage has devoted little discussion to the becoming of infrastructure (Stuedahl et al. 2016), and to which infrastructuring strategies this becoming of entails. In this paper, we employ reflexive analysis of Fusion and EUscreen to explore possible strategies for collective infrastructuring, and interrogate how infrastructures and the conditions that surround them can be instrumental in constructing and contributing to cultural commons. Presenting insights and findings from the collaborative design efforts made for the infrastructures in question, the paper thus addresses the complexity and limits of infrastructuring for cultural commons.

The article begins by bringing together the concepts central to discussions on commons, cultural commons, infrastructures, and infrastructuring from the selected literature so as to illuminate and contextualize the empirical work done in the two design cases that form the foundation of our study. Against this backdrop we then discuss and reflexively analyse some of the infrastructuring strategies and design solutions deployed in these projects. In doing this we make use of the concepts of installed base and gateway that have been developed within studies on infrastructures. We conclude our exploration with a discussion of the need for more flexible, open-ended and commons-like approaches in infrastructural design.

\section{The backdrop: cultural commons and infrastructuring}

Interdisciplinary research on commons is rooted in the study of shared natural resources and of the people and social dynamics involved with them. This research tradition has documented several ways in which communities formed around a set of common-pool resources (CPR) - traditionally being linked to forests, fisheries and/or land - have developed institutions and means of collectively governing them (Ostrom 1990). Commons arrangements are thus ways of caring for and sometimes earning a living from a CPR in a sustainable manner without depleting those resources. The particular arrangements for each commons are unique, but in general they fall outside better-known private or public-property strategies. Because governing a successful and sustainable CPR is not a straightforward endeavour, commons (according to this research tradition) are described as a governance regime 
for shared resources that are vulnerable to social dilemmas (Ostrom and Hess 2007). Potential problems - social dilemmas - with these natural commons are often located in the use, and especially the overuse/over consumption, of shared resources (Hardin 1968).

Since the mid-1990s, scholars have proposed that the 'information commons' and the 'knowledge commons' emerging in the digital age are as relevant a subject of study as the more traditional natural commons (Hess 2008; Frischmann et al. 2014). Information commons have often been characterized as intangible or immaterial. In these types of commons, the social dilemmas and challenges are also associated with congestion and free-riding, but the dilemma of scarcity or depletion - typical of natural CPRs - works in different ways. It is argued that these types of new commons follow a logic of abundance and non-rivalry, rather than scarcity, but that they are nonetheless susceptible to new types of enclosure and commodification (Ostrom and Hess 2007; Boyle 2008).

The origin of the discussion about cultural commons in the digital and networked era can be traced back to two ideas about particular freedoms related to culture, and specifically to cultural re-use and appropriation. The first argument is built around the right to access and appropriate knowledge and culture that are, or should be, common. Lessig's $(2002$ 2004) writings on free culture emphasize that people should have the right and the tools to create and build upon the found digital culture, including on the Internet, and that people should be able to share their new derivative and creative works. This argument became one of the foundations for initiatives such as Creative Commons (CC) that offer a partial design solution in the form of a licensing framework and tools that could enable people to share and create new cultural production on more flexible terms than the existing intellectual-property regimes (IPR) allowed. Frameworks like CC can potentially support people in pooling creative digital resources to achieve common benefits, and eventually create digital commons. Benkler has summarized this argument as follows: "If we are to make this culture our own, render it legible, and make it into a new platform for our needs and conversations today, we must find a way to cut, paste, and remix present culture" (Benkler 2006).

The second argument is built around the need to safeguard common resources from commodification. For example, Boyle (2003) propounds a narrative of enclosure, and discusses the threats of privatization and commodification that are confronting many common digital goods and resources today. According to Boyle this "second enclosure movement", e.g. the withdrawal or fencing off of information and digital cultural artefacts, is made possible by new technologies and mechanisms available online. Later on, Boyle (2008) has also discussed battles over intellectual property, and how the current regimes are harmful to our culture, creativity and innovation. When he discusses the "networked commons of the mind", he argues that the problems do not lie in rivalry over commons, but in other collective-action dilemmas, such as the lack of incentives and motivation to create common resources to begin with (Boyle 2008). Along the same lines as Boyle, but with a different 
vocabulary, Hyde (2010) defends cultural commons by developing an argument and a plea for our common culture, emphasizing how knowledge is common to all and should be safeguarded from commercial interests.

The use and development of the concept of 'cultural commons' in scholarly literature are recent. In addition to the more philosophical perspectives put forward by Lessig (2004), Boyle (2003'2008) and Hyde (2010) as discussed above, there are also several more empirically grounded studies on cultural commons that have focused on the institutional arrangements surrounding them, and have investigated these as arenas of collective action (Bertacchini et al. 2012; Madison et al. 2010). The term 'cultural commons' has also been utilized as a political vehicle or strategic tool for pursuing change in the cultural sector (for examples see e.g. (Edson 2015; Edwards and Escande 2015). In addition, cultural commons have become a favoured concept for discussing how ordinary people can take part in the processes and practices of culture institutions. Hence, cultural and memory institutions are increasingly exploring ways of creating new partnerships with their publics and of opening up the possibilities for people to curate, collect, contextualize and create cultural works from their digital collections (see e.g. Ridge 2014). It should also be mentioned that, already a decade ago, "participation culture" was being used as shorthand to refer to new modes of media practices and production that rely on social networks, participation platforms and common-pool like resources on the Internet (see e.g. Jenkins 2006; Benkler 2006).

While cultural commons is indeed quite a broad concept, in this paper we focus on the cultural resources that both cultural and memory institutions are responsible for preserving and creating access to (including the practices related to them), and the cultural materials that people create in their everyday life. From this perspective "cultural commons" are evolving commons, cumulative in nature, sites where various positioned groups and individuals negotiate the value, creation, use and governance of diverse cultural resources. These groups not only shape our common cultural heritage and memory, but also create common-pool resources and knowledge commons.

It is important to recognize that institutions' common-pool resources of digital cultural heritage, and their management, differ significantly from commons-based peer production and participation systems online. On the one hand, in this case, the official institutional processes of digitization, archiving and preservation are often guided by rigorously defined best practices, policies and standards (e.g. the EBUCore metadata standard for audiovisual collections based on Dublin Core). On the other hand, the governance and management of common resources relies more on evolving social practices co-developed by community members in more flexible and fluid processes. A well-known example of this flexible process is the community-created folksonomies that emerge through the use of the hash-tag symbol (\#) on Twitter, and which add additional context and metadata, and make the navigation and organization of content easier. Also, on YouTube there are everevolving social practices for e.g. initiating and sustaining audiovisual dialogue 
between community members, developing new genres, and the creation of workarounds for annotating and manipulating video (Wesch 2008; Botero et al. 2010).

Because of these differences in approach, it is often the case that digital cultural heritage resources shared and maintained by institutions are associated with infrastructures and practices (both technological and social) other than those in and through which everyday users and communities engage. There are, of course, some initiatives emerging that aim to build bridges between these two sides. First, from an institutional perspective is worth mentioning Flickr Commons, an online repository that provides open, free access to digital images whose copyrights have expired or are unknown. Flickr Commons allows the general public to use published images without restrictions, and also provides a means for people to enrich the photographs by adding comments or other metadata on the platform. Flickr Commons has grown into an open, online catalogue of historical and archival images contributed by a variety of memory institutions, having started as a pilot project in which one institution collaborated with Flickr. The second example derives from the strong Wikimedia community, with the GLAM-Wiki initiative having created concrete practices and formed partnerships with memory institutions that are interested in sharing their offerings on Wikipedia and Wikimedia. These collaborations have taken multiple forms. For example, the Wikimedia community has improved online articles about some of the participating institutions' objects and collections (through the Wikipedian in Residence program); alternative cultural heritage repositories have been collectively developed (e.g. Wiki Loves Monuments); and the community has also developed specific software tools to aid memory institutions (e.g. to make batch uploads to Wikimedia via the GLAMwiki Tools project). Through initiatives like these, institutions and communities alike are attempting to contribute to global cultural commons. In these two examples the platforms used have first served people and their collaborative efforts (e.g. media sharing), and only later, when user volumes have grown and novel media practices have become more established, have digital cultural heritage institutions entered the platforms. There are also other recent efforts, such as Europeana Labs, that attempt to bring memory institutions' own platforms and practices together with community practices and community-created content and software.

In most cases, unfortunately, memory institutions pool their collections and offerings for open access, but do not pay sustained attention to people's actual media practices. It is also common for institutions not to offer the means for people to take part in decision-making or governance of the emerging cultural commons. It can also be the case, when commercial platforms are involved, that the future sustainability of the efforts becomes uncertain, and that co-optation threatens the harvested resources. Weaving connections to overcome the constraints that keep institutions' and communities' platforms and practices apart, and thus limit the emergence of truly cultural commons, is still a big challenge. What issues and processes should be brought into consideration when the particular conditions for contributing to cultural commons 
are taken seriously? These remain underdeveloped areas of research, which we aim to address in this article.

In design studies, the concepts of common, commons, and engagement with the commons literature have been of particular interest for the Participatory Design approach (Björgvinsson 2014; Seravalli 2012; Teli 2015). Participatory Design (PD) practices share commitments and foundations with the commons literature, for example, they both build upon the capabilities and rights of people to act, negotiate, and decide on their own futures; both traditions discuss the potentials and dilemmas of collective action; and in both traditions there is an interest in understanding which types of infrastructures could support collective action (Marttila et al. 2014). Moreover, in line with PD's political commitment, an argument has been made that PD should seek to align its efforts with and contribute to digital infrastructures and spaces that specifically nurture commons (Teli 2015; Hakken et al. 2015). In order to pursue this interest in nurturing and sustaining commons, we will next review the relationship between infrastructure and infrastructuring, and clarify what our cases can tell us about engaging in infrastructuring for cultural commons.

Infrastructures are often defined as common-purpose structures that are designed and built to support human action, a sort of assemblage that carries and transports other things, in the way that a road, a bridge or a cable does. Most of the time, when these assemblages are "finished", they turn into a sort of substrate that disappears into the background. Taking as a starting point previous work on the growing importance of information infrastructures as an integral part of contemporary life (Star and Ruhleder 1994; Neumann and Star 1996; Star and Bowker 2002), the argument has been made that we should consider infrastructures more in their ongoing, relational terms. Scholars in traditions such as Science and Technology Studies (STS) and Information Systems studies (IS) have proposed that infrastructures are not merely substrates that disappear or things that are built and then left behind, instead infrastructures are constantly in the process of change and becoming (Neumann and Star 1996; Star and Bowker 2002; Karasti 2014) and therefore it is critical to trace backwards and forwards the relations that are created between people, materials and structures at all levels (Star and Ruhleder 1996). To trace the implications of this relational view more accurately, Star and Bowker (2002) suggested that it is more interesting to ask 'when' something is being perceived as an infrastructure by its users, rather than 'what' an infrastructure is.

These long-term, relational preoccupations have led to a renewed interest in the doing and making of infrastructures, in what has been called "infrastructuring". Infrastructuring can be considered as a framework for thinking about design activities in more emergent terms (Karasti and Syrjänen 2004). This approach to design stresses that, instead of focusing solely on particular artefacts and neglecting the surroundings in which the artefacts are placed, it is specifically the surroundings that become of critical concern (Pipek and Syrjänen 2006; Pipek and Wulf 2009). As characterized by Star and Ruhleder (1996), and further elaborated by (Pipek and 
Wulf 2009), information infrastructures are always rooted in social and technological infrastructures. This means that there is what these authors term an installed base for any given infrastructure. This installed base can be technological, but it can also consist of social arrangements and practices. Given the presence of an installed base, any new part added or new whole contributed will inherit some strengths and weaknesses from that base. According to Neumann and Star (1996), infrastructure building is also different from building other self-contained systems, in that doing infrastructuring requires linking many communities (already rooted in their own installed bases) into a larger network. Infrastructuring work thus requires that this larger network be made into an active participant, something that in turn inevitably results in various technical and social interdependencies.

Accordingly, when doing infrastructuring, a lot of design work turns towards creating a continuous alignment between different communities and between ways of doing things (Björgvinsson et al. 2010; 2012a' b) while accounting for the creative 'design' activities of professional designers and users, without necessarily privileging either view (Karasti and Syrjänen 2004; Pipek and Syrjänen 2006). Empirical research on how infrastructures emerge through infrastructuring has pointed out that such continuousalignment processes are partly mediated by what is called gateways (Jackson et al. 2007). Gateways usually refer to assemblages and technologies that allow linking and bridging otherwise incompatible or disparate socio-technical infrastructures and practices. Most commonly, recognized gateways are technical appliances or interfaces that connect separate systems, networks or programmes together (David and Bunn 1988). However, as pointed out by Edwards et al. (2009) and Jackson et al. (2007), gateways should not be considered solely as technologies, but gateways are also socio-technical solutions. This is illustrated for example by the case of standards, which need to be integrated both into organizations and into the everyday lives of the people who use those standards, and thus develop new practices that act as gateways, too (Edwards et al. 2009). Organizations can also act as gateways and play an important role in mediating between systems and actors (Zimmerman and Finholt 2007). In a similar way to the installed base, a gateway can thus be technological, while it can also be a social arrangement and a practice. From a design point of view gateway-like effects can sometimes be achieved by setting up in-between infrastructures (Botero and SaadSulonen 2010). The concept of an in-between infrastructure denotes interventions and arrangements that enable experimentation among heterogeneous systems and with different actors before an actual infrastructure or its associated future practices have been settled. In-between infrastructures are thus temporary arrangements and sociomaterial assemblies, embedded in and emergent from a collaborative, or at least multiuser/multi-site, context that requires making provisional structures in order to move towards either more complete, more workable, or more formalized infrastructures. They allow those involved to rehearse future practices, and offer possibilities for understanding what the actual infrastructural initiative could require.

Next, we turn to the empirical cases and discuss some insights gained from these experiences, using selected concepts from the infrastructuring and cultural commons 
approaches to give us a sounding board against which to project, reflect, and explore our experiences.

\section{Tales of two infrastructures: Fusion and EUscreen}

The purpose of this paper is to shed light on collaborative infrastructuring strategies in the design and development of infrastructures for digital audiovisual cultural heritage, and to suggest how design can contribute to sustaining and nurturing cultural commons. To elicit our argument, we draw on our past field and design work in the collaborative design and development of two information systems: Fusion (authors 1 and 2) and EUscreen (author 1). Fusion aimed at providing a decentralized software system and digital tools for ordinary people to publish and share community-created media, while EUscreen aimed to create wider public access to already existing, archived European audiovisual digital cultural heritage.

These infrastructures aimed at either 1) providing digital tools to support people's creative activities with, and sharing of, community videos, or/and 2) creating more meaningful and wider public access to, and facilitating the appropriation of, European audiovisual digital cultural heritage. Even if the projects that developed these platforms were different - as we will elaborate later - there were many similarities in the foundations and objectives of these endeavours. The core objective in both cases was to learn from pre-identified user groups' existing media practices, and to connect together and support the evolution of these practices in relation to the digital tools and services developed. The second fundamental aim was to stimulate and support people's emerging creative re-use activities on various levels (ranging from accessing media to social enrichment and video remixing), and to develop openended infrastructures and software capabilities that could be shaped and further appropriated by the general public.

Both platforms were designed for audiovisual collections, although the media files in these collections originated from highly diverse sources. In Fusion the videos were mainly user and community-created, while in EUscreen professionals from various European audiovisual archives and broadcasting corporations curated the subcollections from their holdings. In addition, the platforms aimed to foster different use and creative activities. Fusion was targeted at helping communities of practice to share and produce community-created content in their mediated everyday life, while EUscreen is an access point for professional television programming and audiovisual heritage. Furthermore, the design approach to how to develop an infrastructure differed: in Fusion the starting point was communities and their practices, while in EUscreen the infrastructural development stems from the existing archival audiovisual content in various institutions. Even if these cases are dissimilar, and were carried out in different media and technology landscapes - the plan for Fusion was laid in the same year as YouTube was launched, while EUscreen's first version was made public when multiple video-sharing and editing platforms were emerging online - taken together these two experiences provide a broad view of the 
infrastructuring challenges for digital audiovisual cultural heritage, and point to issues that we propose are central to the emergence of cultural commons across time.

Both of these endeavours were part of long-term research initiatives co-funded by the European Union, and involved multi-professional consortiums from various European countries. The projects evidently had multiple objectives and various research outcomes in different research domains. Our treatment of the specific cases, which follows, focuses more on the participatory design efforts for developing Fusion and EUscreen infrastructures for digital cultural heritage, and less on the more technical software-development issues, although we do give an account of some of these issues. We conduct our reflexive analysis through the lenses of collaborative infrastructuring using two intertwined concepts: Firstly, we aim to locate and bring to the fore some of the abundant installed bases that we have encountered. Secondly, we trace some of the socio-technical gateways and inbetween infrastructures that we have designed or utilized in the projects. Using these concepts we highlight some of the design workarounds and ad-hoc arrangements we have devised to explore both the potentials of the envisioned social-technical systems, and the communities' emerging creative re-use activities and social practices.

These points are discussed and elaborated in the sections to come, however, first we present the cases individually in chronological order, and summarize the research activities, materials and participants.

\subsection{FUSION - support for communities' everyday media practices}

Fusion was an experimental peer-to-peer audiovisual file-sharing system for communities of practice and their creative activities. With Fusion communities were not only able to manage, share and archive their audiovisual productions in a decentralized way, they could also create customized audiovisual social media applications for themselves. The platform was developed as part of a three-year research project called P2P-FUSION ${ }^{2}$. The project had ambitious practical objectives. Firstly, it aimed at providing support for communities' social activities and media practices by encouraging the creative re-use of audiovisual content, specifically collaborative sharing, editing and enriching of videos, and providing tools for this. The second aim was to provide inbuilt software toolkit capabilities that would enable the development of social media applications, or the customization of existing applications to meet the needs of communities' particular practices. Thirdly, the project aspired to foster a conversation about finding solutions to various intellectual property rights (IPR) issues related to copyrights and the legal re-use of audiovisual materials.

\footnotetext{
${ }^{2}$ The Fusion platform was developed during the P2P-FUSION project (2006-2009) co-funded by the EU FP6 Framework program.
} 
In terms of technology, the Fusion platform connected a number of layers: a peerto-peer network for decentralized storage and distribution of media files, a distributed metadata layer with several social-processing and enrichment features (e.g. annotations and recommendations), an embedded licensing procedure for the content that utilized the Creative Commons licensing framework, and Social Media Application ToolKit (SMAK), which included components that end-users could combine to create their own social-media applications (Figure 1). These were Fusion's main software components and were prototyped and used throughout the lifespan of the project, and have been integrated into other systems and infrastructures. The Fusion system itself is, however, not currently available.

The design and development of Fusion were carried out in collaboration with selected user groups and communities (referred as content communities within the project, and hereafter). Our design involvement in the project related mostly to these participatory design efforts, especially those for the SMAK toolkit, as this was Fusion's main interface with end-users. The collaborative design partners, participants and other stakeholders took part in various continuous, programmed interactions (e.g., interviews, design tasks, workshops). Table 1 below provides an overview of the main activities and empirical materials that formed the basis for our research (see Marttila et al. 2011 for a more detailed account of the co-design process for Fusion and SMAK).

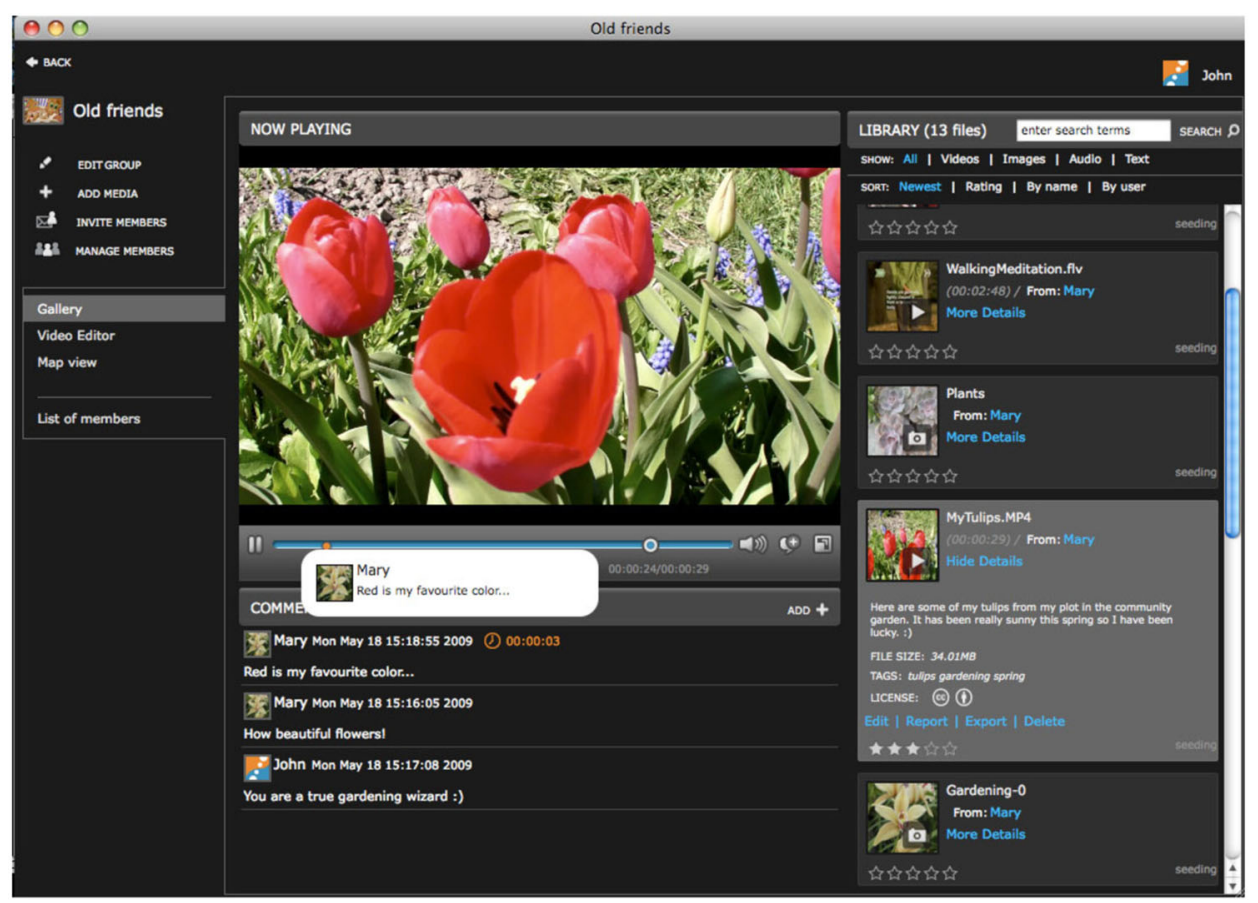

Figure 1. A screen shot of one of Fusion's social media application prototype built with the components from Social Application Toolkit (SMAK). 
Table 1. Overview of the design research and collaborative design activities in the P2P-FUSION project, which were carried out in three different countries: Finland (FI), Hungary (HU), and The Netherlands (NE).

\begin{tabular}{lll}
\hline Activity & $\begin{array}{l}\text { Participants and } \\
\text { collaborators }\end{array}$ & $\begin{array}{l}\text { Design research artefacts/ } \\
\text { interventions }\end{array}$ \\
\hline
\end{tabular}

[A1] Identification and mapping of content communities.

(FI, HU, NE)

[A2] Selection of and invitation to content communities.

(FI, HU, NE)

[A3] Information Days. (3) (FI, HU, NE)

[A4] Local co-design workshops SMAK. ( 15) (FI, HU, NE)

[A5] International co-design workshops SMAK. (2) (HU, FI)

[A6] Developer workshops. (2) (FI)

[A7] Co-design and validation projects (2)

- Education and Archive integration (HU, FI)

[A8] Co-design and validation experiments. (1) - Archive integration. (NE)
Project partners.

15-20 community representatives.

Project partners.

20-30 community members Project partners.

3-5 members from each community (8 communities) Design team: 3-4 members.

3-5 members from each community (3 communities). Design team: 3-4 members.

$\sim 7$ external developers. Fusion developers: 3.

$\sim 25$ students.

4 teachers.

$\sim 18$ youth-club members.

$\sim 2$ youth organizers.

Project members and Fusion design team.

Project members

(+ Archivists and cataloguer)

Fusion developers: 1.
Scoring criteria

Overview data-sheet and selection table

Community web presence

Semi-structured interviews.

Scoring criteria.

Scenarios.

Use cases.

Media Inventories.

SMAK Toys kit (design game).

Paper prototypes.

Mash-up examples.

Scenarios.

Communities' own media.

SMAK Toys kit.

Functional SMAK

prototypes - Community

TV, Jose, Family Archive,

Gallery.

Paper prototypes.

Scenarios.

SMAK Toys kit.

Scenarios.

Fusion code and prototypes.

(+ WebBridge)

Fusion prototype

(+ Publishing server)

Archive Material.

Video Essay assignment.

Interviews.

Questionnaire.

Fusion prototypes and proof of concept. (+Publishing server and SAVI) Archive Material. 
All these events were recorded on audio and/or video, and the resulting design artefacts were documented (e.g. photographs, screen shots). In addition to the materials obtained through these specific co-design engagements, empirical data was collected in the form of field notes that were shared with the whole project consortium. In the case of the SMAK software development, we also made use of the discussions in the issue tracker and in the project-wiki documentation. As Fusion aimed to enable legal creative re-use of audiovisual materials, and of related copyright and intellectual property, the discussions were also collected into a public blog.

\subsubsection{Developing Fusion - co-design partners and participants}

Because there was no pre-existing corresponding technological environment to support the kinds of audiovisual practices that Fusion aimed to support, there were no existing users who could provide insights to aid the design and development of this infrastructure. With no clear, pre-existing user base, the main strategy implemented for the infrastructural development of Fusion was to engage such communities of practice as could benefit from the proposed infrastructure, without those communities necessarily being aware of such needs themselves. Consequently a lot of time and effort was invested in developing a methodology for identifying and inviting potential communities to collaborate in the project. These communities were to be active in some way in producing audiovisual media, and should already be creating, or at least interested in using, audiovisual media so as to document their community activities, to learn from each other, or to communicate with others about their activities. Findings and insights from some of our earlier research on emerging de-centralized audiovisual practices, such as amateur video documentation of skateboarding, also informed the infrastructural development of Fusion. The project was premised on the idea that a decentralized peer-to-peer (P2P) architecture could provide these types of communities with a more efficient and relatively inexpensive environment, one that could also give them control over their content (in terms of both privacy and of ample scope for open media sharing) as opposed to the more prevalent centralized server options that were being developed at the time.

The involvement of the communities as co-design partners was carried out in three phases: First, we conducted a mapping of possible content communities, in order to list promising co-design partners and to obtain a more profound understanding of their current community media content and their practices online. This information was used in the creation of various use cases that Fusion could support. The content communities were identified and selected on the basis of a methodology and of criteria developed by the project partners ${ }^{3}$. The list was initiated with the names of potential communities compiled through snowballing contacts and suggestions from the project partners' own networks in Finland, Hungary and The Netherlands. This list included a wide variety of

\footnotetext{
${ }^{3}$ The content communities selection and engagement in P2P-FUSION was conducted together with Stichting Nederland Kennisland (NE), Budapesti Muszaki es Gazdasagtudomanyi Egyetem (HU) and the University of Art and Design (TAIK), where the authors of this paper worked at the time.
} 
interesting communities, varying from very technologically and socially organized amateur film makers and musicians, activists, senior-citizenship, citizen-journalism and media-education communities to other more informal, small-scale groupings represented by sports circles of snowboarders and skateboarders, music hobbyists, and family or parenting communities, all of whom already had some kind of audiovisual cultural production connected to their community practices [A1]. The list was made more specific and extended by surveying the identified communities' websites and discussion groups. Their interests and characteristics were further mapped, while other potential communities were identified at the same time. The initial list was then reduced to 30 promising communities, with ten communities selected from each country. For each of these 30 communities a standardized overview data-sheet was produced so as to be able to compare them in depth (through phone interviews, sometimes email correspondence, and by consulting their websites). After a thorough comparison had been carried out using a scoring model, the project partners then invited for interview 15 communities that were regarded as viable partners (five from each country) based on their number of members, the stability of the community, and their ability to commit to a co-design process [A2]. These semi-structured interviews aimed to present the general idea of Fusion to community representatives, to obtain a clear view of their ability and willingness to participate in the project, and to begin charting their current audiovisual practices and infrastructures. To kick-start the co-design process a series of information day events were organized in all three countries [A3].

In the second phase of the community engagement we expanded the collaboration with some of the selected communities, who participated in co-design workshops, created scenarios and paper prototypes, and used demos and prototypes of the socialmedia applications built specifically for them with Fusion [4,5,7]. The community involvement closely supported the design process for the Social Media Application ToolKit (SMAK), and through it more indirectly Fusion's other software layers. The communities participating in the co-design of SMAK included: an online music makers' community that supported music and band-driven activities, such as concerts and promotion activities; an extended family, which consisted of geographically separated members, who shared their life events with each other using blogs and photo sharing services to keep the family connected; a volunteer-based folk-dance community that organized activities for children and youths, including traditional dances, singing, drama, play and performances, and who were interested in connecting more audiovisual media to these practices; and two sports-enthusiast communities comprised of acrobatics and parkour practitioners, who trained and organized events, which they documented on video and shared with community members and other communities, or then videos were captured to learn new moves and tricks. In addition, some specific use scenarios were explored with some educational communities and developer communities (e.g. mobile phone application developers) [A6]. Most of these co-design partners were located in Finland, where the design and development of SMAK was also carried out.

In the third and last phase of the co-design process, when a more developed software base already existed, the emphasis was on validating some more advanced and 


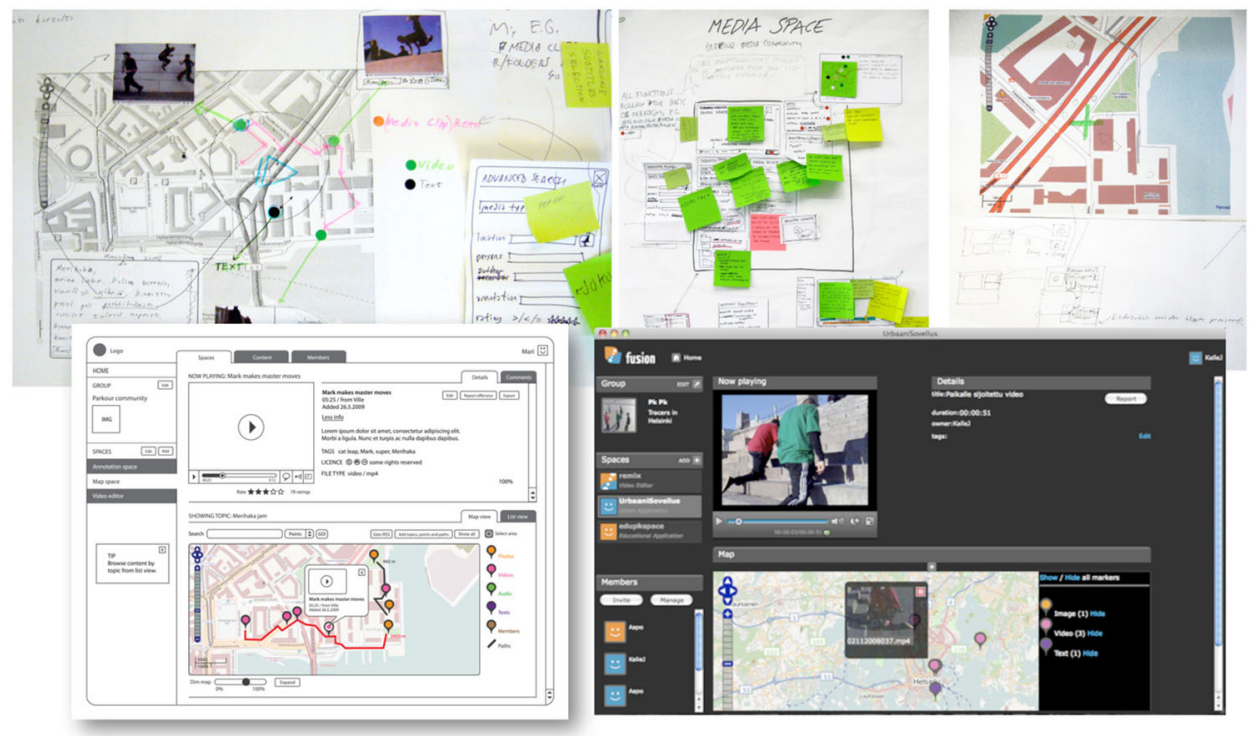

Figure 2. Paper prototypes of the parkour community's application concept, and the Fusion's map application.

specific use scenarios for Fusion via concrete proof-of-concepts (Figure 2). One of these was a model for using Fusion to compile media in educational settings. The second concept aimed to test the feasibility of Fusion as an alternative channel for publishing and distributing media content from memory institutions (e.g. national media archives), and acting as a potential link for feeding community created content into the archives of memory institutions.

These later forms of engagement then included an exploratory project organized in collaboration with teenage students and teachers from a secondary school in Budapest, Hungary, and the National Audiovisual Archives of Hungary. The students tested Fusion by using digital audiovisual heritage content from the NAVA archive in a concrete learning project linked to their curriculum [A7]. Another exploratory project consisted of a series of experiments and proof-of-concept demos carried out by The Netherlands Institute for Sound and Vision, and aimed to understand the implications and potentials of community-created content as new cultural heritage to be included in the archive's collections [A8].

\subsubsection{Understanding community media practices}

Since the content communities were the starting point for the infrastructural development, a lot of effort was invested in understanding the communities, their history, and current and emerging practices, and how these were mediated through audiovisual means. The selection process for the content communities provided the first systematic overview of the practices and the installed base that the Fusion system would inherit. By analysing the data-sheets, interviews and data gathered on information days $[\mathrm{A} 1,2,3]$, we learned about the socio-technological factors present in these 
communities, which were often products of trial-and-error experimentation, rather than a conscious development of mediated practices. These installed bases included e.g. multiple shared email addresses, memory sticks and hard drives, emailing lists, old floppy disks and tapes, mixed with online picture sharing collections. These assemblies of media and tools were not only used as a basic communication infrastructure, but also as informal archival and distribution systems for communities' audiovisual media production and other creative activities. The findings confirmed to those involved in the project that there is a need for software components that communities themselves can customize to better fit various different practices (e.g. in the form of SMAK components). The results of the community mapping also revealed the limitations of the few server-side audiovisual solutions available at the time, and the dependency on hard-to-contact and/or expensive servers, which hindered the communities' capacity to expand their audiovisual practices.

During the co-design sessions $[\mathrm{A} 4,5,7]$ it become evident that the communities' installed base was a result of a creative - but also a very fragile - bricolage of different components and practices. To give an example of such a practice, some of the communities were regulating access to part of their content using simple strategies like acquiring multiple domain names, or using practices such as sharing easyto-guess passwords. Doing the mapping helped some of the communities, as well as the design team, to realize that the communities relied heavily on existing social networks and on the identity they had already formed online. For most of the communities it was essential to be able to integrate any potential new software into their current digital media ecosystem. This was especially the case with their web services (e.g. simple content management systems and blog services), for which they had already created some workflows. Despite the original commitment to P2P solutions, it became evident that Fusion would also need to include ways of bridging the P2P network's contents and functionalities into existing web functionalities, as this was the most common installed base that the communities and their collaborators relied on. This is the backdrop to, and one origin of, the WebBridge application and gateway that is briefly discussed later in this article.

Just as the communities had their own installed base, the Fusion technology platform also relied on an installed base. Fusion was built as an open-source software project; parts of the platform were developed mostly utilizing existing open-source software and experimental $\mathrm{P} 2 \mathrm{P}$ technology components developed prior to the project. At the time of the project, P2P technologies were mostly employed by specialists or "geek" communities, or used in very specific applications (e.g. simple file sharing). This meant that many of the protocols necessary for the new platform were not yet stable enough or standardized, which became a significant burden in the effort to build a generic platform. In particular, the project was unable to overcome Network Address Translation (NAT) traversal problems in the P2P clients. Because NAT methods for P2P varied, and some only used the server when establishing the connection, while others relayed all the data through it all the time, persistent incompatibilities and conflicts ended up adding bandwidth costs, increasing latency, or were detrimental 
to real-time video sharing, and therefore entailed concrete user-experience limitations. Without overcoming this conflict, the benefits that P2P technologies offered these content communities could not be realized in practice. In addition, other technological difficulties, which are beyond the scope of this paper, created restrictions in terms of the limited video formats that the platform could support, the particular standards that could be relied on, and a number of other interoperability issues.

An additional installed base issue that arose here is that of copyrights. The installed base mixes elements of technology and social practices that have created hitches in the project. At times, the licensing support chosen for Fusion conflicted with some of the communities' existing media practices. For example, some community's event documentation used "borrowed" music for which they did not have a license of permission. Another community remixed copyrighted video materials without approval or consent from the copyright holders. Because Fusion aimed at explicitly facilitating legal creative re-use of audiovisual materials, the platform included an easy way to assign a Creative Commons licence that would specify the terms of sharing for all content or media files shared in Fusion. Implementing this mandatory software feature in the system forced users to select a licence for all media content injected into Fusion. However, adding this feature to the system did not necessarily mean that communities changed their media practices and started attributing material to the original sources. Nor did it mean that communities would start licensing their own work or would consider the various licence options, or using the Creative Commons licences outside of Fusion. Paradoxically, even existing public discourse around copyrights and media sharing on the Internet acted as a sort of installed base, and had a major impact on the way people approached Fusion. When Fusion was presented as relying on a P2P network, people often associated the system with "illegal" file sharing, probably because the technology first became known through platforms such as Pirate Bay and Napster.

\subsubsection{Gateways and in-between infrastructures}

The empowering impact that the system was hoped to achieve could not be fully concretized, and the final Fusion system did not fully meet the project objectives. In the following section we reflect on some of the bridges, the many gateways and the in-between infrastructures that we devised, or made use of, around Fusion in order to simulate the envisioned infrastructure and emerging media practices.

Following classic participatory design techniques we created a toy-like design game called SMAK Toys, in order to make visible both the content communities' installed base, and the installed base of the technologies used in Fusion. This SMAK Toys design game made it possible to run meaningful co-design workshops with a shared vocabulary among people unfamiliar with software development. SMAK Toys used magnetic cards (30) to represent different types of media content, and to create different uses and ways of sharing it (Figure 3). During the first workshops [A4] we used SMAK Toys to map communities' current and future media practices. We also co-created scenarios and paper prototypes of specific applications that could support 

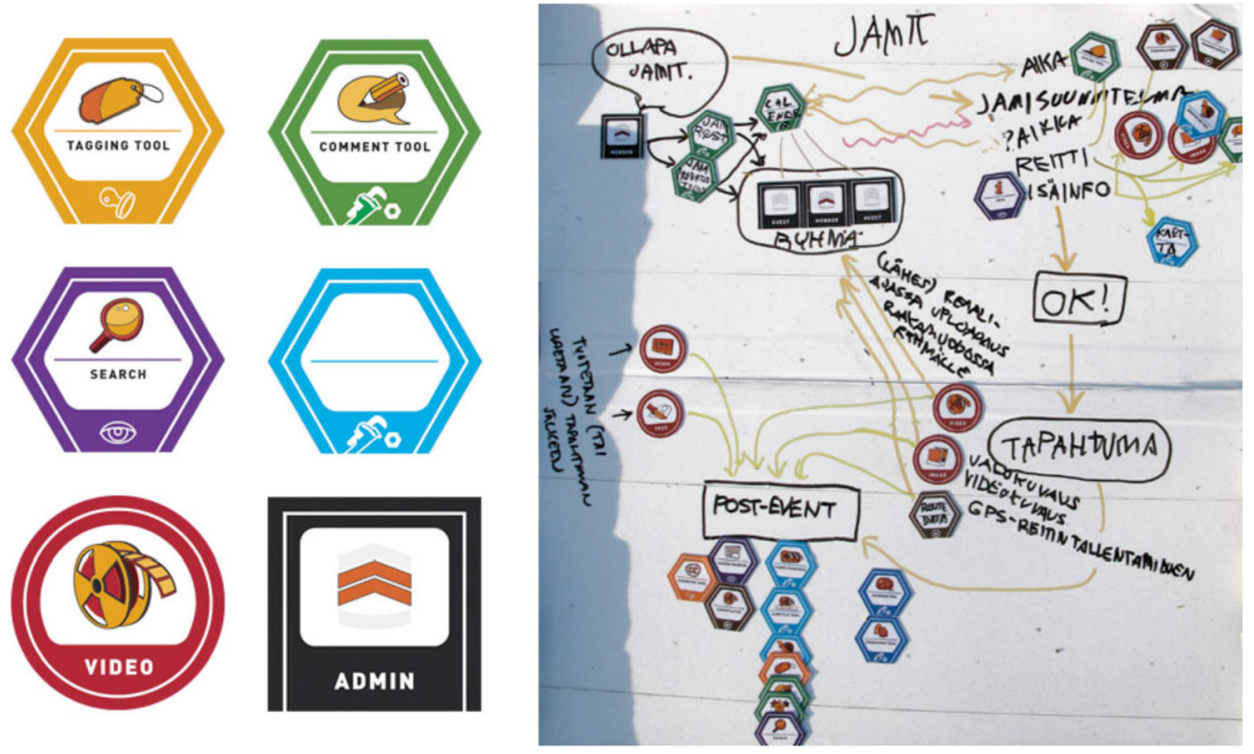

Figure 3. The SMAK Toys cards (left), mapping the media sharing practices in a parkour jam (right).

them (e.g. sharing photos and videos of a community event such as Parkour Jam). The individual cards functioned as 'building blocks' and formed a shared vocabulary that helped to create a common understanding of current media practices (accomplished by probing the communities' current installed base) and possible future media practices (accomplished using forthcoming Fusion social-media-applications concepts). The building blocks helped to make more concrete and visible what the community practices consisted of in detail, as well as to define and make visible the specific characteristics and functions that the new potential applications required (Figure 3).

This design game as an infrastructuring strategy was used at the design-before-use stage (Redström 2008) and aimed at mapping how community practices and the tools they use are connected to other socio-technical systems and materials. Ehn (2008) discusses the configuration of design patterns (building on Alexander 1964) as a way to support user participation, future use and appropriation. In our case, the design patterns operated in two ways: First, for the participating communities, the design patterns provided an analytic, structured and visual representation of their current media practices and installed base, something which they had rarely thought about or articulated to one another. In these sessions community representatives jointly reflected on their practices, and in many cases, after creating the maps and scenarios, community members reflected on their communities' own governance structures, on the power relations that they had not noticed before, and on possible social dilemmas that existed in their cultural production activities when using their current practices and tools. This facilitated articulation and questioning of what they wanted from Fusion's features, and even from their own installed base systems. Secondly, for the designers and software developers, the patterns provided valuable input in terms of recognizing existing and emerging 
media practices, current and possible audiovisual materials, and the communities' concrete installed bases. All this formed the basis for the feature and function specifications for the software components of the Social Media Application ToolKit (SMAK).

Besides using participatory design techniques such as the SMAK Toys design game that served as a gateway to media practices, the project also prototyped several other concrete technical gateways. The first software gateway component, referred to as WebBridge, provided an interface between Fusion and the web, making it easier to share data and media content with other web-based applications or native applications. WebBridge was deemed necessary by many of the co-design partners, as they were not keen on putting a lot of effort into an experimental infrastructure that would not contribute to their online presence. Later, the WebBridge gateway also made it possible to develop the Fusion Mobile application. A second gateway component supported cases involving the integration of current archival infrastructure, with community created content coming from external software systems, into the archive's own catalogue system. This was made possible by conceptual mapping of the Fusion metadata to the archive's metadata formats (in this case the Sound and Vision archive) using a relatively simple custom-built Python script (SAVI). This proof-of-concept provided valuable input for understanding the requirements for the archiving in memory institutions community content that comes from external sources (i.e. What roles, working practices and collaborative arrangements need to exist between communities and archives for such an archival process to function?), and offered ideas about the potential for collaboration between official institutions and online communities (e.g. an archive could get assistance from communities to perform social tagging of archive collections) (Figure 4).

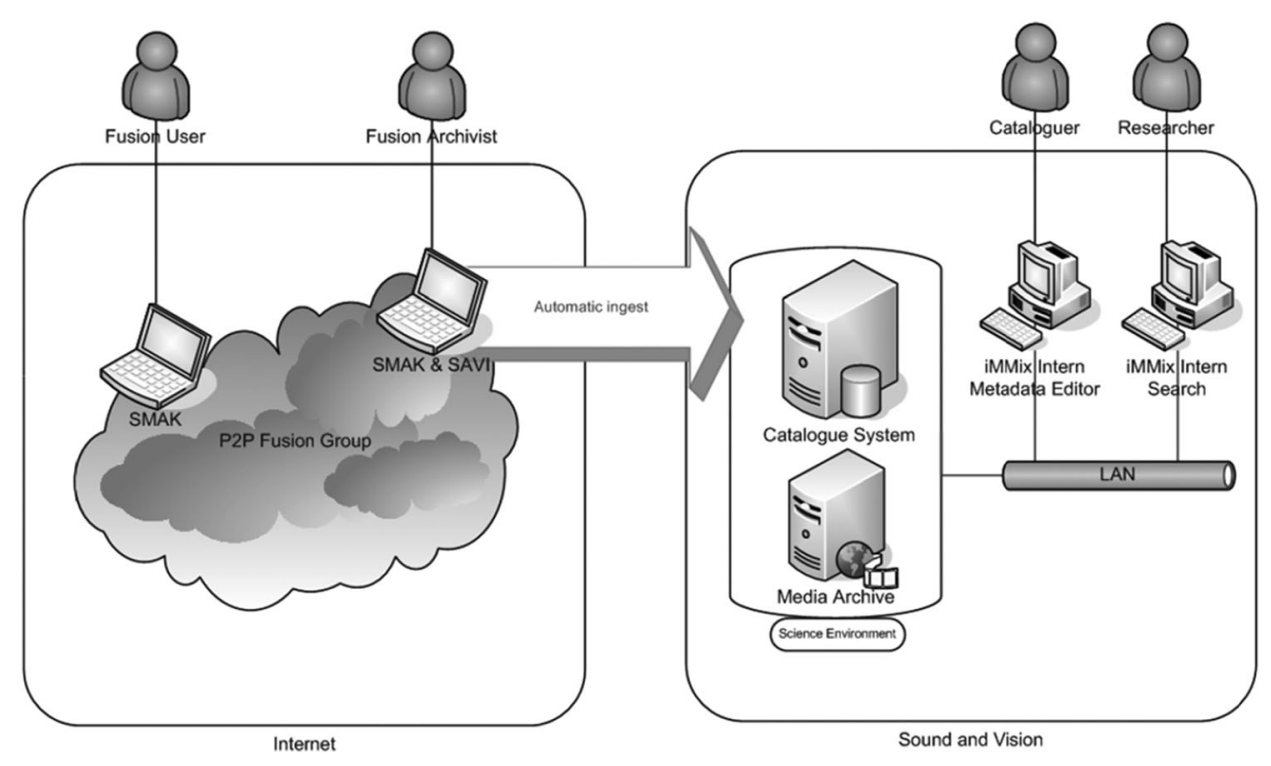

Figure 4. Schematic overview for the proof-of-concept scenario for archiving community content. Source: P2P-FUSION, sound and vision. 
In addition other in-between infrastructure prototypes were built on immature and disparate socio-technical infrastructures to help translate some of the abstract components of Fusion into more concrete forms. First, these in-between infrastructures were produced as paper prototypes, and later as mash-ups using existing software tools (not necessarily based on P2P technologies). In the later phases of the project, we developed alternative functional prototypes to better communicate the envisioned functionalities and infrastructure to the participating communities. When some of the key future components of Fusion were delayed due to technical incompatibilities, we created alternative demos and pilots to enable hands-on experimentation and to obtain more realistic input as regards the adaptability of software toolkits for use in communities' practices (Figure 5 and 6). These were used to simulate and stimulate the becoming of infrastructure, its use and inhabitation.

The project also required a lot of mediation of shared understanding on various levels and between various stakeholders. One concrete example of a gateway created for this purpose was the various common-pool information resources compiled for different actors (e.g. community members and other end-user developers) in the form of wiki-based learning resources.

As discussed in this section the project encountered limitations in the key technologies and community practices that hindered the reach of Fusion and prevented it from being fully used. However, after having participated in the process, most of the communities did emerge from it with a clearer understanding of the place of audiovisual practices in the present and future of their community practices. Some communities, such as the extended family, started producing more video documentation that they then continued to share in an ad-hoc fashion, and others, such as the sports community, started more systematically exploring the use of emerging platforms like YouTube.

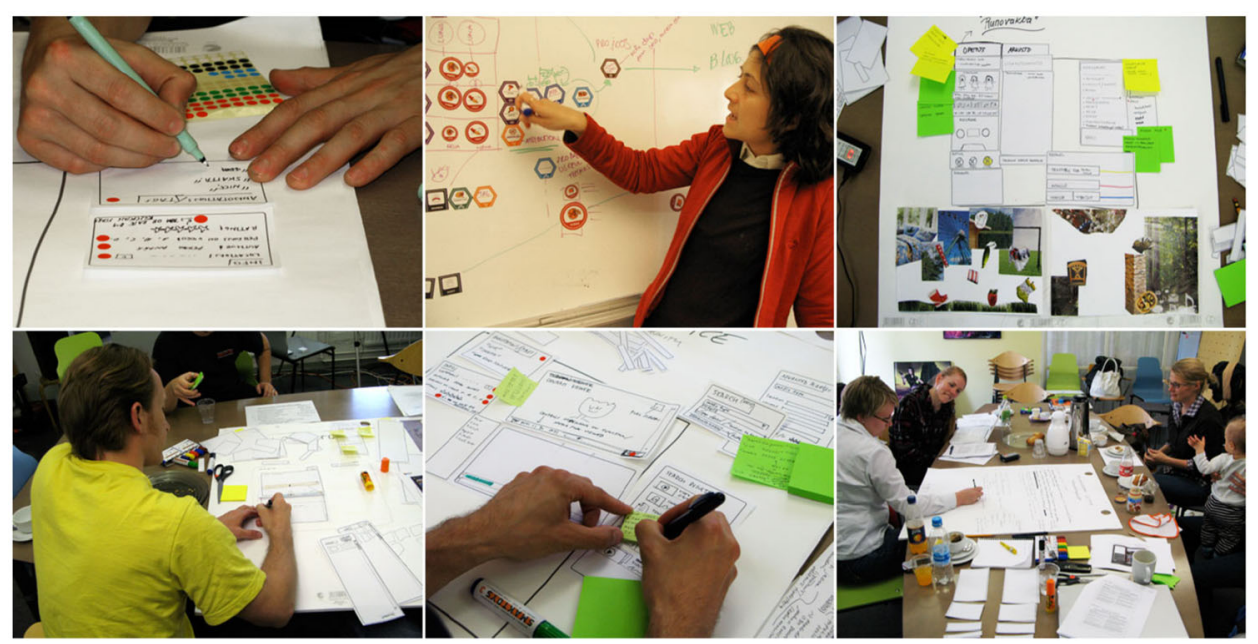

Figure 5. Co-designing community application concepts with SMAK Toys and paper prototypes. 

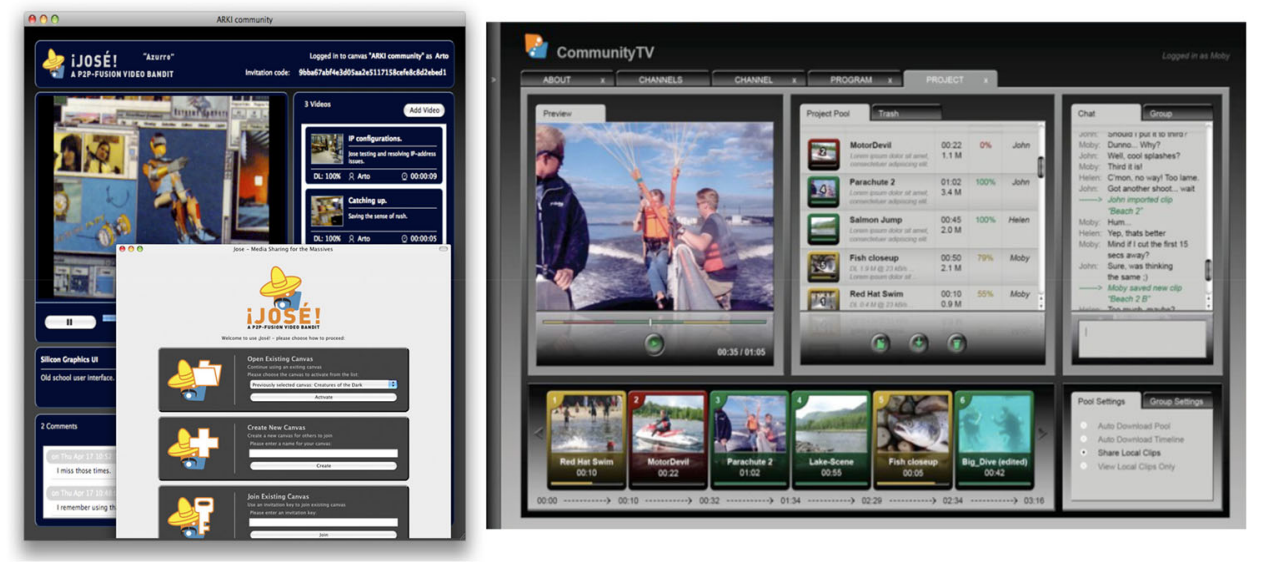

Figure 6. Screen shot of the José pilot application (left) and the community TV pilot application (right) in Fusion.

\subsection{EUSCREEN - access to european audiovisual heritage}

EUscreen.eu is a platform that promotes access to and use of European television programming and audiovisual cultural heritage ${ }^{4}$. The technology platform allows multicultural and multilingual exploration of European audiovisual content, and encourages audience engagement with the offerings ${ }^{5}$. The audiovisual collection made available for EUscreen is curated by archives and national broadcasting corporations across Europe. The portal presents the items grouped under various themes (e.g. Arts and Culture, Conflicts) and genres (e.g. News), and includes broad metadata for the media, such as the broadcast date and geographical coverage (Figure 7).

The EUscreen platform aimed to support, on the one hand, multi-professional collaboration (across memory institutions), and, on the other, the creative audiovisual re-use activities of various user groups. To achieve these aims, the project adopted a collaborative design approach with various stakeholders in three main areas: defining and building a digital online audiovisual collection to populate EUscreen; designing a technology platform and its offerings; and understanding and supporting the emerging practices of creative re-use. Table 2 below summarizes the main design research activities in thematic order. It should be noted that, for the purposes of this paper and for our analysis, we concentrate only on the early design and development work on the

\footnotetext{
${ }^{4}$ The EUscreen portal was developed within two EU-supported projects: the EUscreen project (2009-2012), whose main objective was to design and develop the portal and produce an audiovisual collection of European television history, and the EUscreenXL project (2013-2016), which continued the development work, launched a new responsive interface and enlarged the audiovisual collection.

${ }^{5}$ Currently the EUscreen portal makes publicly available a wide collection of over 60000 items of television programming, and over 1 million metadata records of more than 20 audiovisual archives and public broadcasting companies throughout Europe. Besides being a stand-alone platform, EUscreen is also the largest provider of audiovisual heritage to Europeana.eu (an interface for digitized European cultural heritage providing currently access to over 50 million records of digital culture).
} 

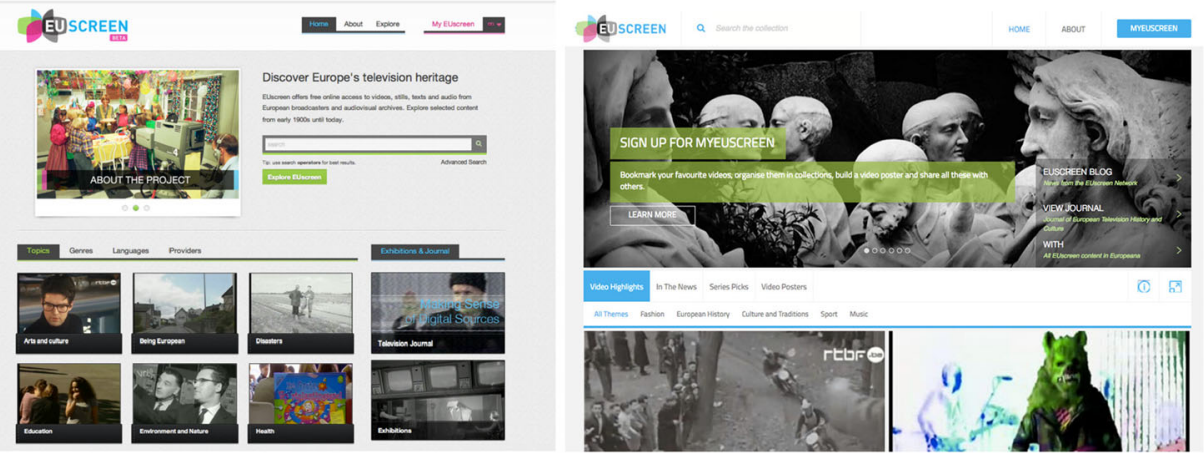

Figure 7. The EUscreen landing page - beta version in October 2014 (left). The current landing page of EUscreen (right).

first beta version of the EUscreen platform ${ }^{6}$, because the key infrastructuring decisions were taken during that time. In terms of the technology, the period of analysis covers the launch of the first public beta version of the platform and various parallel experiments carried out around it.

\subsubsection{Building the foundations for EUscreen - content and prototypes}

From the beginning, the project plan defined four very broad application areas for EUscreen: education, research, leisure and open cultural productions. These themes were already selected, based on previous work and collaboration by some of the consortium partners, when the funding application was made. The first phase of the project thus included refining and concretizing the characteristics and requirements of possible user groups and communities in relation to these thematic areas. Use-case narratives collected from all the project partners' previous experiences formed the bases for the initial functional user requirements made for the front-end development of EUscreen [B1]. Through further engagement with the selected user groups and stakeholders several use scenarios were created in the selected thematic areas [B2]. To validate the relevance of these use scenarios, evaluation sessions were conducted in various European countries, using the same design of testing protocol (including e.g. questionnaires, interviews and assignments) [B3]. Results from this previous process fed into two important activities: first, the audiovisual archives and public broadcasters providing media to the portal used them to create guidelines and criteria for a content selection schema in response to the devised scenarios. The content providers also prepared a set of shared metadata categories that everyone was to work with. Secondly, the project's technical and design partners used the scenario work to guide the design and development of the first version of the platform.

In order to build the audiovisual collection for EUscreen, and to clarify the possibilities for cultural appropriation and creative re-use that the portal was planning

\footnotetext{
${ }^{6}$ A newer version of the portal was launched in October 2014 as part of the work in EUscreenXL. Since that project ended, EUscreen has been maintained through a foundation structure.
} 
Table 2. The EUscreen project's collaborative design activities and participants.

$\begin{array}{ll}\text { Activity } \quad \text { Participants and collaborators } & \begin{array}{l}\text { Design research artefacts/ } \\ \text { interventions }\end{array}\end{array}$

[B1] Definition of user groups and mapping of initial user requirements.

[B2] Scenario Framing.

[B3] Scenario evaluation and testing in real-world settings.

[B4] Mapping possible content and understanding archives' existing intellectual property and rights situations.

[B5] Mapping of open audiovisual content.

[B6] Co-design workshops (3).

[B7] Collection design

(AV content selection).

[B8] Collection experiments and testing (AV content selection)

[B9] Co-design and testing workshops (2)

[B10] Open cultural production workshops (3) a. License to Remix, b. Make Open Video, c. Linking Media Workshop.

Project partners.
Various invited collaborators.
12 Field experts.
$\sim 8$ selected future stakeholders
(e.g. teachers).

38 participants (from 7 different countries during one month).

Project partners.

17 respondents from audiovisual archives and public broadcasters. Project partners.

5 representatives from archives and public broadcasters.

8-40 participants.

2 Researchers.

Project partners

(audiovisual archives and public broadcasters).

Project partners.

(audiovisual archives

and public broadcasters)

$\sim 10$ selected

future stakeholders.

12-15 Students.

3 Researchers.

a. 11 young adults/3 external experts/2 video \& VJ practitioners.

b. 10 designers, developers and artists.
c. 30 Students/1 teacher
2 Researchers.

Pre-existing knowledge.

Initial functional

requirements.

Use and user scenarios around research and leisure/ cultural heritage and open cultural production.

Semi-structured interviews.

7 Future-use scenarios.

Online survey design

and results.

Audiovisual (AV) content samples.

Structured interviews.

AV content samples.

EUscreen Wireframes.

Paper prototypes.

Visualizations.

Future-use scenarios.

Copyrights/terms of use clearance.

Content-selection guidelines.

AV content samples.

Open Images prototype.

Virtual Exhibition prototype.

Content selection guidelines.

AV Content samples.

EUscreen Portal.

Virtual Exhibition builder prototype.

Thematic Virtual Exhibitions.

Rehearsing practice exercises. AV content samples.

Mash-ups and video remixes. Audiovisual compilations. Interviews.

to offer its users, a survey of intellectual property rights was carried out with the content providers [B4]. The survey included questions on each organization's 
content selection and the copyright-clearance process. This helped us understand the scope of the rights and the terms of use of the material made available for EUscreen. Importantly it also investigated what kinds of creative use activities future EUscreen visitors could actually do with the audiovisual content on the portal (See Marttila and Hyyppä 2014a) for a detailed account of the survey and its design implications for EUscreen). As it turned out that a large part of the available content had lots of copyright limitations, many of the envisioned use scenarios were impossible to realize. Some of the responding institutions that showed an interest in and possibilities for releasing materials for EUscreen under more flexible and open terms were invited for further interviews and exploration [B5].

With the first working versions of the portal available, and a clearer idea of the concrete possibilities for supporting creative re-use with future EUscreen content, several co-design workshops were held to develop the main features, and to continue testing the concepts and scenarios developed [B6,7]. Due to the limitations caused by the copyright restrictions on the audiovisual collections that the project encountered in the first phase, the portal development did not encompass the more ambitious use and re-use functions (e.g. creating video playlists, extracting parts of videos as quotations, creating derivative works) envisioned in the advanced scenarios with the communities. The resulting EUscreen portal ${ }^{7}$. thus mostly had some core functionalities (e.g. searching, viewing items on a video player, and displaying metadata for each item from the collection) and limited content. To overcome these drawbacks, and in order to deepen our understanding of the challenges involved in creative re-use of online archival audiovisual materials, the project adopted a practical, design oriented perspective that included designing experiments, making alternative prototypes and staging events with a view to informing future EUscreen developments in more varied directions. To experiment and pilot open distribution of audiovisual heritage, a branded section was created for EUscreen on a separate hosting platform, Open Images, where five invited institutions released a selection from their video collections under a flexible Creative Commons licence [B8]. We also created an experimental prototype for EUscreen that allowed the combining of EUscreen content from different institutional sources and time periods. This prototype was called the Virtual Exhibitions (VE) builder, and as the name suggests it was a tool for curating and creating virtual exhibitions to be displayed on the portal. The prototype was designed and developed in close collaboration with user groups and participant institutions [B8,9].

In the last co-design phase we engaged with domain experts, practitioners, and selected user groups representing the fields of research, learning, leisure and open cultural production. All of them were deemed to be potential beneficiaries from using online archival audiovisual content and creative tools of the type that EUscreen could have in the future. In the same vein, all the exchanges provided opportunities to

\footnotetext{
${ }^{7}$ The EUscreen portal was designed by Aalto University (concept design and interaction design) and Noterik, a Dutch software development company specialized in audiovisual media.
} 
identify ways in which future EUscreen developments could enable and support emerging creative activities, as well as supporting the advanced digital archiving of video. Three case studies centred on open cultural-production practices were conducted [B10]: A License to Remix! - a participatory video remix workshop - studied current and emerging audiovisual remix and mash-up practices among young people via participant observation and interviews. One of the central objectives was to understand the challenges involved in legal remixing, and to find ways that EUscreen could enable and support this. (For more details of the study see (Marttila and Hyyppä 2014b). The Open Video Make workshop invited advanced users and experts to create experimental interactive videos that went beyond traditional remixing by including open data and other not so well explored video features. Our aim was to study how practitioners were making use of temporal and spatial video characteristics and metadata, and how to support expert users within EUscreen. The Linking Media educational workshop was held in a high school as a part of a history course. The workshop aimed to study how students could learn about and deepen their knowledge of European history by creating rich audiovisual compilations that linked cultural heritage material to other sources, and by sharing these collections and stories online with others.

\subsubsection{Building on the installed base}

The installed base in EUscreen was heterogeneous and complex. Even when we set aside the various technology systems that it aimed to combine - an inventory would not serve the purposes of this paper - the installed base was truly a melting pot of different professional worlds and work practices combining archival practices, software design and everyday media practices, to name but a few.

Nevertheless, the installed bases that ended up having the most weight in the project were those related to content, since the foundations of the project were very much built on the audiovisual materials of the participating institutions. The EUscreen collections originate from several archives and public broadcasting corporations, and they therefore also inherit the installed bases of those institutions and collections. These included a wide variety of curatorial processes, collection management policies, multi-lingual and multi-cultural issues whose relevance varied according to the collection, a myriad of different metadata standards, incompatible classification schemas, and multiple video formats to deal with. These challenges were expected, since the project's key objective was to achieve an interoperable collection of television heritage, both in standardizing the metadata, as well as in harmonizing the complex copyright issues. In fact, this attempt to increase interoperability and improve access to European audiovisual heritage was already bootstrapped in another EU-funded project prior to EUscreen, whose history became part of this new future infrastructure (e.g. differences in work practices, subcollections and formats were already identified as being important to overcome).

However, from the start it was the dissimilarity of copyrights and terms of use that posed the biggest challenge to the development of the socio-technical infrastructure. 
As reported above, intellectual property issues, much more than the broad rhetoric of supporting creativity that underlies the project, clearly defined the final content selection for the EUscreen portal. Two examples illuminate this dilemma well: First, due to IP regulations and the challenges faced by the institution in clearing the rights, most of the video items available belong to the genre of news and current affairs, as these materials were often produced by the contributing institutions themselves, and consequently the rights to release them in EUscreen were easy to secure. While this type of content may be valuable to many user groups, it should be noted that many other types of interesting and useful content were excluded. These factors limited the scope of the heritage available on the platform, and its creative re-use value for the communities. Second, even though the content was enriched by detailed metadata, audiovisual content often requires other types of contextualization, or an engaging narrative, to be interesting or to become relevant. Again, the copyright restrictions on the EUscreen collection limited the possibilities for creating audiovisual stories that would combine materials from different sources and time periods - a very simple, basic use case that most people expected to be able to achieve with this type of content. It is also interesting to bear in mind, however obvious it is, that the consortium agreement and other legal contracts also added a strong layer to the foundations of the infrastructure.

\subsubsection{Gateways and in-between infrastructures}

To overcome the limitations imposed by the IPR restrictions, and to demonstrate the value of emerging media practices and creative re-use of audiovisual heritage, the project partners developed workarounds, demos and experiments in collaboration with representatives from the targeted user groups. For these, several pilots, codesign workshops and experiments were carried out in the form of gateways and inbetween infrastructures.

A key in-between infrastructure developed to try to bypass some of the limitations of EUscreen's installed base was the creation of the Virtual Exhibition builder (VE) prototype (Figure 8). The prototype allowed the combination of the portal's audiovisual content and text to curate and create video exhibitions around a selected theme or topic. Using the VE builder it was possible for public broadcasters and audiovisual archives to curate their content and create a thematic sub-collection, with contextualization and a possible narrative, without actually joining or editing the clips together. The VE builder worked as an in-between infrastructure that connected parts of the audiovisual collection, its content-management system, and the software platform, and provided an interface for creating virtual exhibitions that were then presented on the actual EUscreen platform. As is often the case with gateway projects, the aim of the VE builder was to "hide" the complex infrastructure from the curators, and to create a bridge between practices, systems and different programmes.

The prototype was created in a co-design process together with selected representatives from the institutions taking part in the project as media content providers. Designing the VE builder and tools involved various activities, such as workshops, in 


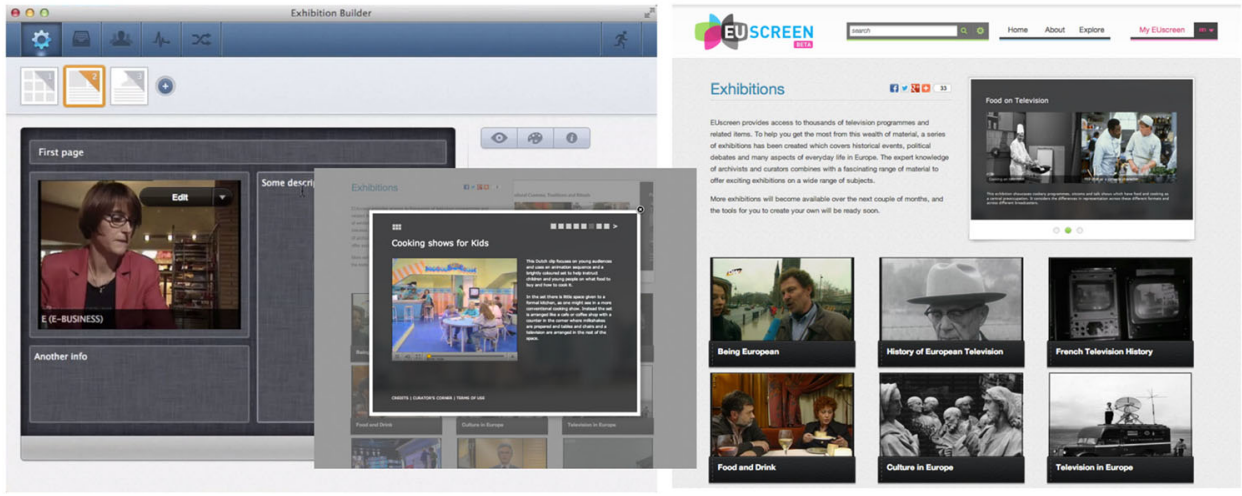

Figure 8. Screen shot of the Virtual Exhibition builder prototype (left) and the exhibition page (right) on the EUscreen portal.

which collaborative design methods were used to generate ideas, and to inform and improve the design [B6]. The VE builder prototype was tested with all the content providers in another workshop in order to mirror the design in the institutions' needs, and to obtain feedback on the usability of the tools. Some of the organizations also created virtual exhibitions from the selected highlights from their own collections. Additionally, some researchers tested the prototype and created their own thematic exhibitions to communicate audiovisual stories about historical topics or events. This prototype was also used in a workshop with students, who experimented with the VE builder to test the applicability of such a tool in educational settings. Unfortunately this prototype, though functioning, was not made available for public use due to the copyright restrictions (Marttila and Hyyppä 2014a).

Since it was not possible to allow downloading of the materials on EUscreen, in order to explore the more open and creative re-use scenarios developed for the portal, the project decided to make an experimental prototype on another existing platform for video hosting and sharing (Open Images) ${ }^{8}$. The EUscreen project created a separate branded section there, displaying content from a few of the EUscreen content providers who released their media under a Creative Commons licence. This workaround solution and simulation of the particular use scenario downloading video for creative re-use - gave the involved archives a practical, positive experience of what it entailed to make their content open, what to take into account, and what to expect from open releases. For example, some very basic prejudices were overcome when none of the released content was used in ways that the institutions would have found non-respectful. But choosing the Open Images platform to showcase the open EUscreen content had one drawback, this time not in terms of copyright, but in terms of the quality of the

\footnotetext{
${ }^{8}$ Open Images is a Dutch initiative by Nederlands Instituut voor Beeld en Geluid together with Kennisland. For more information and to access the platform visit: http://www.openimages.eu/.
} 
downloadable video; the low quality and the variety of video formats made re-use for new cultural works cumbersome.

The open culture workshops proved to be an interesting way to rehearse creative reuse practices, as well as to demonstrate the value of such practices in the context of the EUscreen project. They stimulated discussion around the topic within the consortium and with user groups, and provided valuable insights into what new requirements these emerging media practices place on socio-technical infrastructures. Two of the participatory workshops were held in collaboration with a local youth centre and a highschool, and the third was organized in conjunction with a larger festival run by the Open Knowledge movement. Besides bringing in new user groups to discuss these matters with us, these collaborating organizations played an important role in legitimatizing the activity and the approach to the rest of the consortium. Sometimes, the collaboration also created expectations that were difficult to manage and to meet, for example, in the case of the high-school teachers who were keen on continuing the collaboration and using in their courses the EUscreen tools that were ultimately not published.

\section{From silos to commons?}

Through reflexive analysis of the empirical cases of Fusion and EUscreen we have addressed two interrelated concepts that are central to infrastructuring processes: the installed base and the gateway. These notions were useful in discussing the sociotechnical infrastructural development required for digital audiovisual cultural heritage, and for identifying infrastructuring strategies that could contribute to cultural commons. In the following, we summarize some of the key findings and infrastructuring strategies found from these cases.

\subsection{Probing the installed base}

A growing infrastructure inherits both the limitations and capabilities of the installed base. Due to the heterogeneous, evolving nature of a growing infrastructure, it is important to actively probe and reveal the possible connections and interdependencies between different infrastructural elements, resources and actors. As discussed in the treatment of the cases, in both Fusion and EUscreen the installed base significantly influenced the design and development work on the systems. A lot of time was invested in the infrastructuring work on two levels: reconfiguring and redesigning the connection points with and between actors and resources, and on the integration - or simulation - of these different parts. In Fusion, particular attention was paid to the communities and their practices, and to connecting them with some of the institutional practices and processes. As these practices differed greatly, it was a learning process for both sides. The application concepts and technological offerings were shaped according to the needs and wishes of the communities. In turn, in EUscreen the foundation for infrastructural development lay more in the institutional processes and the audiovisual content deriving from the institutions. If we adopt the 
understanding of infrastructuring as a long-term relational process, contributing to the emergence of cultural commons cannot be done by relying solely on the technological or social aspects. Instead many other actors, resources and processes have to be identified and brought together in a strategy that considers content, communities and the technologies that can weave them together.

\subsection{Stimulating and simulating use through gateways}

The experiences gained from the cases considered here also demonstrate the importance of creating gateways and in-between infrastructures to bridge otherwise incompatible socio-technical infrastructures and practices. This reflects the special attention that needs to be paid when "bridging the gap between technology development and appropriation" (Pipek and Wulf 2009, p. 467). In both cases, however, for different reasons, creating so-called workarounds, demos and pilot projects became an essential infrastructuring strategy. In Fusion, the prototypes and software pilots were called for in order to simulate and stimulate future uses and practices (this includes both the creative re-use of media and end-user development), because the technology development was hindered by the limitations encountered in key technologies, challenges to connecting technology layers, and other interoperability issues. In EUscreen, the creative workarounds and infrastructuring activities were mainly called for by the legal and intellectual property issues (e.g. legal contracts, privacy issues and copyrights) related to the platform and to the audiovisual collection. In both cases, current and emerging media practices were rehearsed with various user groups and stakeholders through in-between infrastructures and assemblies of patched configurations of existing software tools and audiovisual media content.

An important infrastructuring strategy in relation to gateways in both projects was bridging between silos of the common-pool resources and practices connected with them. As discussed earlier in this article, there is a gap between the digital cultural heritage collections and the systems that are made accessible by the cultural and memory institutions, and the platforms through which people use, create and produce digital cultural works. By configuring situations and events, such as a participatory video remix workshop for young people, rehearsing the creation of virtual exhibitions with museum professionals, or creating the conditions for open audiovisual platforms, people and institutions together envisioned what an alternative infrastructure could be if there were no current technical or legal constraints. Weaving together contexts, social practices and language, often from significantly different work and media practices, is an important part of the work done to achieve the socio-technical infrastructures that could support cultural commons, and which we aimed to contribute to. In our cases the pilot applications and ad-hoc workarounds enabled the advancement of particular pieces of the infrastructure, but more importantly they supported the process of evolving the practices, and sometimes even the values and changing 
attitudes, of the people taking part in the building of the infrastructure. Even if all of the qualities addressed and explored by the projects' in-between infrastructures and gateways were not implemented in the information systems, there is a good chance that they will be a part of the installed base of other similar infrastructures in the future, and hopefully contribute to creating awareness of the need for cultural commons.

\subsection{Terms of use}

In both cases the legal frameworks had a major impact on the development of the infrastructures. Both in Fusion and in EUscreen rights and terms of use were embedded in all the layers, starting from the Open Source software utilized. In EUscreen the audiovisual collections provided by the institutions had various copyrights and other rights issues attached to them, while in Fusion the project tried to promote an explicit sharing and attribution approach for the community-created content (via the Creative Commons licences). It became evident that copyrights, even when fairly liberal, shape the design, media materials and infrastructure practices in profound ways, thus presenting a practical challenge to the emergence of cultural commons. Communities making use of the offerings of these infrastructures have to continuously assess the legality of their actions on various levels, e.g. archival content, software building blocks, source code, as well as the platform's possible terms of use. Rarely are people invited and included in the process of shaping these rules of participation. Even in EUscreen and Fusion, which were designed in projects with a collaborative mindset, these terms were drafted by legal experts and implemented by designers and software developers. In the infrastructural development it is pertinent to ask: Who should decide how people interact, appropriate and innovate using common cultural heritage and history?

The key to understanding information infrastructures for digital cultural heritage, access to them and their use is addressing the diversity of the characteristics and mechanisms that can contribute to cultural commons. Commons are often thought of as a governance structure and process in which a specific set of rules is in place. However, rather than being explicitly defined and stated, these rules tend to arise from social practices and interactions among people connected to particular resources. In both of the cases reviewed here, the boundaries of common-pool resources and rules-in-use were defined, and sometimes even dictated, by the project partners. Even if the rules were partly formal and party informal, the formal social agreements (license agreement, consortium agreement) permeated the interactions between human and non-human actors. One of the key findings of the established commons-research tradition is that a rich and very specific set of rules has been in use in resilient commons over a long period of time. These rules were well matched to local needs and conditions, and people using and sustaining the commons had a 
chance to negotiate these rules (Hess and Ostrom 2007, p. 7). The cases and experiences discussed in this article indicate that, if in the co-design of information infrastructures we would like to move towards more flexible, open-ended and commons-like approaches, then we need to direct more design attention and infrastructuring activities towards negotiating the governance regimes and terms of participation with all the stakeholders. Professional designers should reflect more on whether and how they impose their own values, politics, and attitudes through codesign and infrastructuring activities; as well as how these concerns are reflected in the design process, and in the emerging infrastructure.

In the case of EUscreen, the designers and software developers encountered resistance from the memory and cultural institutions that were providing audiovisual materials for the platform. During the development of the portal, the designers felt that some representatives from these institutions were reluctant to share authority or control over the materials. This hindered the participation and creative re-use activities. Along with complicated rights issues related to the released content, other challenges for collaboration might be at play. This observation is supported in the literature by a study mapping the factors hindering cultural heritage institutions' opening their descriptive metadata on cultural objects (Verwayen et al. 2011), and by a survey that studied the status quo for digitized and open cultural heritage materials, and the attitudes of cultural and memory institutions (Estermann 2015). However, when instances of concrete collaboration could be rehearsed in secure settings within the projects, everybody recognized the value and potential that existed here.

In the case of Fusion, the software development failed to attract end-user developers. Ironically, this might have been the result of the different layers of Fusion becoming isolated systems, centrally designed in different research institutions and controlled by individual developers. Even if the developed software is Open Source on all levels, commons-based activities and collective action becomes difficult if so-called end-user developers do not have a long-term investment in the infrastructure. In addition, there was little motivation or incentive for content communities to create common resources that would span different communities, even if the project had sought communities with similar interests in different countries. One problematic issue thus became that the co-design and infrastructuring efforts concentrated more on understanding communities' media practices and on translating them into design language, and not so much on facilitating potential developer communities or on tapping into existing common-pool software resources, before the software development (and the financed project) was in the final stage.

\subsection{Rules of participation}

Participating in and contributing to knowledge or cultural commons are often voluntary (as in the Wikimedia Commons already mentioned). In both of our cases, people participated in the co-design activities, e.g. in the hands-on workshops and hacking sessions on a voluntary basis. Sometimes tensions can arise if some people 
are contributing without compensation on a voluntary basis, while others participate as part of their daily job (e.g. those employed by project partners or collaborating institutions, such as school teachers). Especially in the context of Fusion's co-design process there was an imbalance between how much time and effort the participants invested, and what concrete and intangible benefits participating community members gained from the process. In infrastructural development there should be an emphasis on collectively negotiating and articulating the terms of participation and on explicitly discussing everyone's expectations.

Common-pool digital cultural heritage resources are often selected and pooled by institutions or emerge through the processes of inclusion/exclusion that they tend to control, consequently the rules of participation end-up being dictated by those same institutions. A key point is questioning and paying attention to how these cultural materials are designated as 'cultural heritage' in the first place, as this is an originating site of definition and potential conflict, long-term friction, and shifting perceptions, where infrastructures are difficult to pinpoint and have mainly evolved in closed terms. Because of this, infrastructuring processes deeply influence the notion of the commons, and to whom it belongs, to whom its rules apply, and who gets to participate in setting those rules.

The relationship between different local and global needs is also relevant in our cases, as locally and nationally created and nurtured collections of media (both institutional and everyday arrangements) are subject to third-party commercial interests and commodification when pooled for a global, open infrastructure, and further subject to terms/rules of use that people can not influence. Interestingly, in the context of European digital cultural heritage, the EU has recently made significant efforts - through policy guidelines and channelling of funding, from which both of the cases discussed here benefited - to support monetization of this cultural commons globally, and creating new business models and opportunities for sustainability. Commons has often been considered an alternative or as complementary to the dichotomy between market and state, and yet the EU Commission's strategy for public cultural heritage seems to be directed at commercial sustainability, rather than at sustainable cultural commons.

\section{Concluding remarks}

In this article we have reflected on our participation in the design and development of two information systems: Fusion and EUscreen. These two infrastructural initiatives were aimed at contributing, from different angles, to wider public access to, and appropriation of, European audiovisual cultural heritage and digital culture in general. Through the notions of installed base and gateway we have shed light on some strategies for infrastructural development for digital audiovisual cultural heritage, and in the process discussed how design can contribute to sustainable cultural commons. We believe that engagement with the notion of installed base is a useful move for identifying and reviewing in depth what are the components of existing infrastructures and their characteristics, and how they are brought into being and put 
to use. Probing an installed base can also reveal what conflicts and contradictions can be inherited in it, and in turn, contribute to the design contexts at hand. Active probing and understanding of an infrastructure's installed base can be a useful device for the design and development process, and can be helpful for better identifying infrastructural challenges.

The concept of gateway can be used as a practical infrastructuring strategy that helps to identify incompatible socio-technical infrastructures and practices, and identify any design workarounds, interventions and pilots necessary to bridge them, and to stimulate and simulate these new configurations. Learning from our cases we argue that initiatives that aim to support the revitalization of cultural heritage through digitization should focus more on the collaborative dimensions that their platforms and infrastructures are aimed at. Our experiences point to the importance of building bridges between different actors and resources, as well as weaving together different contexts and practices as potential infrastructuring activities that could be beneficial to all involved stakeholders. In addition, building on our cases we argue that creating socio-technical workarounds, ad-hoc arrangements and prototypes in order to simulate and stimulate the current and emerging practices is valuable for infrastructural development. These explorations have a specific role in enhancing technologies and practices, and further, in these staged instances, stakeholders have a possibility to collaboratively create common ground and build shared resources. If adequate, flexible gateways can be proposed during the infrastructural development, more cultural commons can arise.

Despite the limitations of these projects, experiences in Fusion and EUscreen reveal glimpses of the potential that exists when fostering a productive, collaborative relationship between institutionalized digital cultural heritage preservation initiatives and more amateur and peer-to-peer online media practices and infrastructures. Digital cultural heritage infrastructures, when not only concerned with preservation of and creating access to digital cultural heritage, can become catalysts in the construction of shared cultural resources that also enable collaboration between diverse audiences.

Doing the work of participatory, collaborative infrastructuring leads to, or makes visible, discussions of and frictions over what is useful and necessary in a given context, and what can be considered cultural commons and for whom. If digital cultural heritage initiatives take the collaborative dimensions of infrastructuring more seriously into consideration, then the likelihood of nurturing sustainable cultural commons improves. We hope our work is a contribution to that.

\section{Acknowledgements}

We are grateful to the co-design partners and participating communities both in Fusion and EUscreen. We also wish to thank the project partners in P2P-FUSION, EUscreen and EUscreenXL for the fruitful collaboration, and special thanks to our colleagues Kati Hyyppä, Anne Luotonen, Lauri Kainulainen and Kari-Hans 
Kommonen. The anonymous referees have contributed with knowledgeable suggestions and helpful comments of this paper for which we are very grateful.

Open Access This article is distributed under the terms of the Creative Commons Attribution 4.0 International License (http://creativecommons.org/licenses/by/4.0/), which permits unrestricted use, distribution, and reproduction in any medium, provided you give appropriate credit to the original author(s) and the source, provide a link to the Creative Commons license, and indicate if changes were made.

\section{References}

Hess, Charlotte (2012). Constructing a New Research Agenda for Cultural Commons. In E. E. Bertacchini Bertacchini; G. Bravo; M. Marrelli; and W. Santagata (eds): Cultural Commons A New Perspective on the Production and Evolution of Cultures. Cheltenham: Edward Elgar Publishing, pp. 19-35.

Alexander, Christopher W. (1964). Notes on the Synthesis of the Form. Cambridge, Mass.: Harvard University Press.

Anderson, David (2013). Preserving Europe's digital cultural heritage: a legal perspective. New Review of Information Networking, vol. 18, no. 1, pp. 16-39.

Bauwens, Michel (2009). Class and capital in peer production. Capital \& Class, vol. 33, no. 1, pp. 121-141.

Bellini, Francesco; Antonella Passani; Francesca Spagnoli; David Crombie; and George Ioannidis (2014). MAXICULTURE: Assessing the Impact of EU Projects in the Digital Cultural Heritage Domain. In Digital Heritage. Progress in Cultural Heritaage: Documentation, Preservation, and Protection - 5th International Conference, EuroMed 2014, Limassol, Cyprus, November 3-8, 2014. Proceedings. Lecture Notes in Computer Science 8740, Springer 2014, pp. 364-373.

Benkler, Yochai (2006). The wealth of networks: How social production transforms markets and freedom. New Haven: Yale University Press.

Benkler, Yochai (2013). Commons and Growth: The Essential Role of Open Commons in Market Economies. The University of Chicago Law Review, vol. 80, no. 3, pp. 1499-1555.

Bertacchini, Enrico; Giangiacomo Bravo; Massimo Marrelli; and Walter Santagata (eds.) (2012). Cultural commons: a new perspective on the production and evolution of cultures. Cheltenham: Edward Elgar Publishing.

Björgvinsson, Erling (2014). The Making of Cultural Commons: Nasty Old Film Distribution and Funding. In P. Ehn, E. M. Nilsson and R. Topgaard (eds): Making Futures: Marginal Notes on Innovation, Design, and Democracy. Cambridge, Mass: MIT Press, pp. 187-225.

Björgvinsson, Erling; Pelle Ehn; and Per-Anders Hillgren (2010). Participatory design and democratizing innovation. In: PDC 2011: Proceedings of the 11th Biennial Participatory Design Conference, New York: ACM Press, pp. 41-50.

Björgvinsson, Erling; Pelle Ehn; and Per-Anders Hillgren (2012a). Agonistic participatory design: working with marginalised social movements. CoDesign: International Journal of CoCreation in Design and the Arts, vol. 8, no. 2-3, pp. 127-144.

Björgvinsson, Erling; Pelle Ehn; and Per-Anders Hillgren (2012b). Design Things and Design Thinking: Contemporary Participatory Design Challenges. Design Issues, vol. 28, no. 3, pp. 101-116.

Bollier, David; and Silke Helfrich (eds.) (2012). The wealth of the commons: a world beyond market and state. Amherst, MA: Levellers Press. 
Botero, Andrea; and Joanna Saad-Sulonen. (2010). Enhancing citizenship: the role of in-between infrastructures. In PDC 2010: Proceedings of the 11th Biennial Participatory Design Conference. New York: ACM Press, pp. 81-90.

Botero, Andrea; Kari-Hans Kommonen; and Sanna Marttila (2010). Expanding design space: Designin-use activities and strategies. In Proceedings of the DRS Conference on Design and Complexity.

Boyle, James (2003). The second enclosure movement and the construction of the public domain. Law and Contemporary Problems, vol. 66, no. 1/2, pp. 33-74.

Boyle, James (2008). The public domain: Enclosing the commons of the mind. New Haven: Yale University Press.

Bruns, Axel (2008). Blogs, Wikipedia, Second Life, and Beyond: From production to produsage. New York, etc.: Peter Lang.

David, Paul A; and Julie Ann Bunn (1988). The economics of gateway technologies and network evolution: Lessons from electricity supply history. Information Economics and Policy, vol. 3, no. 2, pp. 165-202.

Edson, Michael (2015). Fire and Frost: The Virtues of Treating Museums, Libraries and Archives as Commons. In D. Bollier and S. Helfrich (eds.): Patterns of Commoning. Commons Strategy Group and Off the Common Press.

Edwards, Louise; and Aubery Escande (2015). White Paper European Cultural Commons. Europeana. http:// pro.europeana.eu/files/Europeana_Professional/Projects/Project_list/Europeana_Version3/Milestones/ Ev3\%20MS20\%20Cultural\%20Commons\%20White\%20Paper.pdf. Accessed 10 March 2016.

Edwards, Paul N; Geoffrey C. Bowker; Steven J. Jackson; and Robin Williams (2009). Introduction: an agenda for infrastructure studies. Journal of the Association for Information Systems, vol. 10, no. 5, pp. 364-374.

Ehn, Pelle (2008). Participation in design things. In PDC 2008: Proceedings of the tenth anniversary conference on participatory design Bloomington, Indiana, 1-4 October 2008. New York: ACM Press, pp. 92-101.

Estermann, Beat (2015). Data File and Documentation, Public Use: OpenGLAM Benchmark Survey 2014 2015. Bern University of Applied Sciences, in cooperation with Open Knowledge's OpenGLAM Working Group. https://outreach.wikimedia.org/wiki/GLAM/OpenGLAM_Benchmark_Survey. Accessed 10 April 2016.

Estermann, Beat; Maarten Brinkerink; Gemma Echevarria; Dorota Kawęcka; Sanna Marttila; Joris Pekel; Mariana Valente (2015). Questionnaire of the OpenGLAM Benchmark Survey 2014-2015 (Version 2, Spring 2015). Bern University of Applied Sciences, in cooperation with Open Knowledge's OpenGLAM Working Group. http://survey.openglam.ch/questionnaire_en_v2_EUR.pdf. Accessed 10 April 2016.

Frischmann, Brett M; Michael J. Madison; and Katherine J. Strandburg (2014). Governing Knowledge Commons. New York: Oxford University Press.

Hakken, David; Maurizio Teli; and Barbara Andrews (2015). Beyond Capital: Values, Commons, Computing, and the Search for a Viable Future. New York: Routledge.

Hardin, Garrett (1968). The Tragedy of the Commons. Science, vol. 162, no. 3859, pp. 1243-1248.

Hess, Charlotte (2008). Mapping the new commons. Available at SSRN 1356835 https://ssrn.com/ abstract $=1356835$.

Hess, Charlotte; and Elinor Ostrom (2007). Introduction: An Overview of the Knowledge Commons. In E. Ostrom; and C. Hess (eds): Understanding Knowledge as a Commons. From Theory to Practice. Cambridge, Mass: The MIT Press, pp. 3-26.

Hyde, Lewis (2010). Common as air: Revolution, art, and ownership. New York: Farrar, Straus and Giroux.

Jackson, Steven J.; Paul N. Edwards; Geoffrey C. Bowker; and Cory P. Knobel (2007). Understanding infrastructure: History, heuristics and cyberinfrastructure policy. First Monday, vol. 12, no. 6.

Jenkins, Henry (2006). Convergence culture: Where old and new media collide. New York: NYU Press. 
Karasti, Helena (2014). Infrastructuring in participatory design. In PDC 2014: Proceedings of the 13th Participatory Design Conference: Research Papers, Volume 1. New York: ACM Press, pp. 141-150).

Karasti, Helena; and Anna-Liisa Syrjänen (2004). Artful infrastructuring in two cases of community PD. In PDC 2004: Proceedings of the eighth conference on Participatory design: Artful integration: interweaving media, materials and practices, Volume 1. New York: ACM Press, pp. $20-30$.

Lessig, Lawrence (2002). The future of ideas: The fate of the commons in a connected world. Vintage.

Lessig, Lawrence (2004). Free culture: How big media uses technology and the law to lock down culture and control creativity. New York: Penguin.

Madison, Michael; Brett Frischmann; and Katherine Strandburg (2010). Constructing Commons in the Cultural Environment. Cornell Law Review, vol. 95, no. 4, pp. 657-709.

Marttila, Sanna; and Kati Hyyppä (2014a). Practices and challenges in creative re-use of audiovisual media. In Proceedings of the CUMULUS Conference, May 2014, pp. 301-315.

Marttila, Sanna; and Kati Hyyppä. (2014b). Rights to remember? how copyrights complicate media design. In Proceedings of the 8th Nordic Conference on Human-Computer Interaction: Fun, Fast, Foundational. ACM, pp. 481-490.

Marttila, Sanna; Kati Hyyppä; and Kari-Hans Kommonen (2011). Co-Design of a Software Toolkit for Media Practices: P2P-Fusion Case Study. In J. Pierson, E. Mante-Meijer, \& E. Loos (Eds.): New Media Technologies and User Empowerment. New York: Peter Lang.

Marttila, Sanna; Andrea Botero; and Joanna Saad-Sulonen (2014). Towards commons design in participatory design. In PDC 2014: Proceedings of the 13th Participatory Design Conference: Short Papers, Industry Cases, Workshop Descriptions, Doctoral Consortium papers, and Keynote abstracts, Volume 2. ACM Press, pp. 9-12.

Neumann, Laura J.; and Susan Leigh Star (1996). Making infrastructure: The dream of a common language. In J. Blomberg, F. Kensing and E. Dykstra-Erickson (eds): PDC 1996: Proceedings of Participatory Design Conference. Palo Alto, CA: Computer Professionals for Social Responsibility, pp. 231-240.

Ostrom, Elinor (1990). Governing the Commons: The Evolution of Institutions for Collective Action. Cambridge: Cambridge University Press.

Ostrom, Elinor; and Charlotte Hess (2007). A Framework for Analyzing the Knowledge Commons. In C. Hess and E. Ostrom (Eds.), Understanding Knowledge as a Commons. From Theory to Practice. Cambridge, Mass: MIT Press, pp. 41-82.

Pipek, Volkmar; and Anna-Liisa Syrjänen (2006). Infrastructuring as capturing in-situ design. In 7th Mediterranean Conference on Information Systems, Venice, Italy.

Pipek, Volkmar; and Volker Wulf (2009). Infrastructuring: Toward an integrated perspective on the design and use of information technology. Journal of the Association for Information Systems, vol. 10, no. 5, pp. 447-473.

Redström, Johan (2008). RE: Definitions of use. Design Studies, vol. 29, no. 4, pp. 410-423.

Ridge, Mia (ed.) (2014). Crowdsourcing our cultural heritage. London: Ashgate Publishing, Ltd.

Seravalli, Anna (2012). Infrastructuring for opening production, from participatory design to participatory making? In PDC 2012: Proceedings of the 12th Participatory Design Conference: Exploratory Papers, Workshop Descriptions, Industry Cases, Volume 2. New York: ACM Press, pp. 53-56.

Sotirova, Kalina; Juliana Peneva; Stanislav Ivanov; Rositza Doneva, and Milena Dobreva (2012). Digitization of Cultural Heritage - Standards, Institutions, Initiatives. In J. Peneva; S. Ivanov; K. Sotirova; R. Doneva, and M. Dobreva (Eds): Access to Digital Cultural Heritage: Innovative Applications of Automated Metadata Generation. Plodvid: Plovdiv University Publishing House "Paisii Hilendarski", pp. 25-67.

Stainforth, Elizabeth (2016). From museum to memory institution: the politics of European culture online. Museum \& Society, vol. 14, no. 2, pp. 323-337. 
Star, Susan Leigh; and Karen Ruhleder (1996). Steps toward an ecology of infrastructure: Design and access for large information spaces. Information Systems Research, vol. 7, no. 1, pp. 111-134.

Star, Susan Leigh; and Geoffrey Bowker (2002). How to infrastructure. In L. A. Lievrouw \& S. Livingstone (Eds.): Handbook of New Media: Social Shaping and Social Consequences of ICTs. Sage Publications, pp. 151-162.

Stroeker, Natasha; and René Vogels (2014). Survey Report on Digitisation in European Cultural Heritage Institutions 2014. ENUMERATE Thematic Network January 2014. http:// www.enumerate.eu/fileadmin/ENUMERATE/documents/ENUMERATE-Digitisation-Survey2014.pdf. Accessed 10 April 2016.

Stuedahl, Dagny; Mari Runardotter; and Christina Mörtberg (2016). Attachments to Participatory Digital Infrastructures in the Cultural Heritage Sector. Science \& Technology Studies, vol. 29, no. 4, pp. 50-69.

Teli, Maurizio (2015). Computing and the common: hints of a new utopia in participatory design. In Proceedings of The Fifth Decennial Aarhus Conference on Critical Alternatives. Aarhus: Aarhus University Press, pp. 17-20.

Terras, Melissa (2015). Opening Access to collections: the making and using of open digitised cultural content. Online Information Review, vol. 39, no. 5, pp. 733-752.

Tsolis, Dimitrios; Spyros Sioutas; Michalis Nik Xenos; and Georgios Styliaras. G. (2011). Copyright and IPR management for cultural heritage digital content in peer-to-peer networks. Journal of Cultural Heritage, vol. 12, no. 4, pp. 466-475.

Verwayen, Harry; Martijn Arnoldus; and Peter B. Kaufman (2011). The problem of the yellow milkmaid. A business model perspective on open metadata. Den Haag: Europeana. http://pro.europeana.eu/files/ Europeana_Professional/Publications/Whitepaper_2-The_Yellow_Milkmaid.pdf. Accessed 10 March $201 \overline{6}$.

Wesch, Michael (2008). An anthropological introduction to YouTube. Recording of a lecture titled "The Anthropology of YouTube," given by Michael Wesch on June 23. In 2008 at the Library of Congress, Washington, DC. http://hdl.handle.net/2097/6520. Accessed 10 March 2016.

Zimmerman, Ann; and Thomas A. Finholt (2007). Growing an infrastructure: The role of gateway organizations in cultivating new communities of users. In GROUP 2007: Proceedings of the 2007 International ACM Conference on Supporting Group Work. New York: ACM Press, pp. 239-248. 